\title{
MRSI-BASED MOLECULAR IMAGING OF THERAPY RESPONSE TO TEMOZOLOMIDE IN PRECLINICAL GLIOBLASTOMA USING SOURCE ANALYSIS
}

\begin{tabular}{|c|c|}
\hline Journal: & NMR in Biomedicine \\
\hline Manuscript ID & NBM-15-0279.R1 \\
\hline Wiley - Manuscript type: & Research Article \\
\hline Date Submitted by the Author: & 14-Feb-2016 \\
\hline Complete List of Authors: & $\begin{array}{l}\text { Delgado-Goñi, Teresa; Universitat Autònoma de Barcelona, Departament } \\
\text { de Bioquímica i Biologia Molecular; Universitat Autònoma de Barcelona, } \\
\text { Networking Research Center on Bioengineering, Biomaterials and } \\
\text { Nanomedicine (CIBER-BBN) } \\
\text { Ortega-Martorell, Sandra; Liverpool John Moores University, Department of } \\
\text { Mathematics and Statistics; Universitat Autònoma de Barcelona, } \\
\text { Networking Research Center on Bioengineering, Biomaterials and } \\
\text { Nanomedicine (CIBER-BBN) } \\
\text { Ciezka, Magdalena; Universitat Autònoma de Barcelona, Department de } \\
\text { Bioquímica i Biologia Molecular; Universitat Autònoma de Barcelona, } \\
\text { Networking Research Center on Bioengineering, Biomaterials and } \\
\text { Nanomedicine (CIBER-BBN); Universitat Autònoma de Barcelona, Institut } \\
\text { de Biotecnologia i de Biomedicina } \\
\text { Olier, Ivan; The University of Manchester, Manchester Institute of } \\
\text { Biotechnology } \\
\text { Candiota, Ana Paula; Universitat Autònoma de Barcelona, Networking } \\
\text { Research Center on Bioengineering, Biomaterials and Nanomedicine } \\
\text { (CIBER-BBN); Universitat Autònoma de Barcelona, Department de } \\
\text { Bioquímica i Biologia Molecular ; Universitat Autònoma de Barcelona, } \\
\text { Institut de Biotecnologia i de Biomedicina, } \\
\text { Julià-Sapé, Margarida; Universitat Autònoma de Barcelona, Networking } \\
\text { Research Center on Bioengineering, Biomaterials and Nanomedicine } \\
\text { (CIBER-BBN); Universitat Autònoma de Barcelona, Departament de } \\
\text { Bioquímica i Biologia Molecular; Universitat Autònoma de Barcelona, } \\
\text { Institut de Biotecnologia i de Biomedicina } \\
\text { Fernandez, Francisco; Departament de Medicina i Cirurgia Animals, } \\
\text { Universitat Autònoma de Barcelona } \\
\text { Pumarola, Martí; Departament de Medicina i Cirurgia Animals, Universitat } \\
\text { Autònoma de Barcelona } \\
\text { Lisboa, Paulo; Liverpool John Moores University, Department of } \\
\text { Mathematics and Statistics } \\
\text { Arus, Carles; Universitat Autònoma de Barcelona, Bioquímica i Biologia } \\
\text { Molecular; Universitat Autònoma de Barcelona, Networking Research } \\
\text { Center on Bioengineering, Biomaterials and Nanomedicine (CIBER-BBN); } \\
\text { Universitat Autònoma de Barcelona, Institut de Biotecnologia i de } \\
\text { Biomedicina }\end{array}$ \\
\hline
\end{tabular}


MRSI-BASED MOLECULAR IMAGING OF

\title{
THERAPY RESPONSE TO TEMOZOLOMIDE IN
}

\section{PRECLINICAL GLIOBLASTOMA USING SOURCE}

\author{
ANALYSIS \\ Delgado-Goñi T, Ortega-Martorell S, Ciezka M, \\ Olier I, Candiota AP, Julià-Sapé M, Fernández F, \\ Pumarola M, Lisboa PJ, Arús C.
}

The methodology described in this work is able to characterize the response to $\mathrm{TMZ}$ in preclinical GB both at a defined time-point and in real time, along the therapy protocol. The response patterns detected correlate well with the histopathological analysis of control and treated tumors, at each stage. This strategy has translational potential for monitoring patient response to treatment.

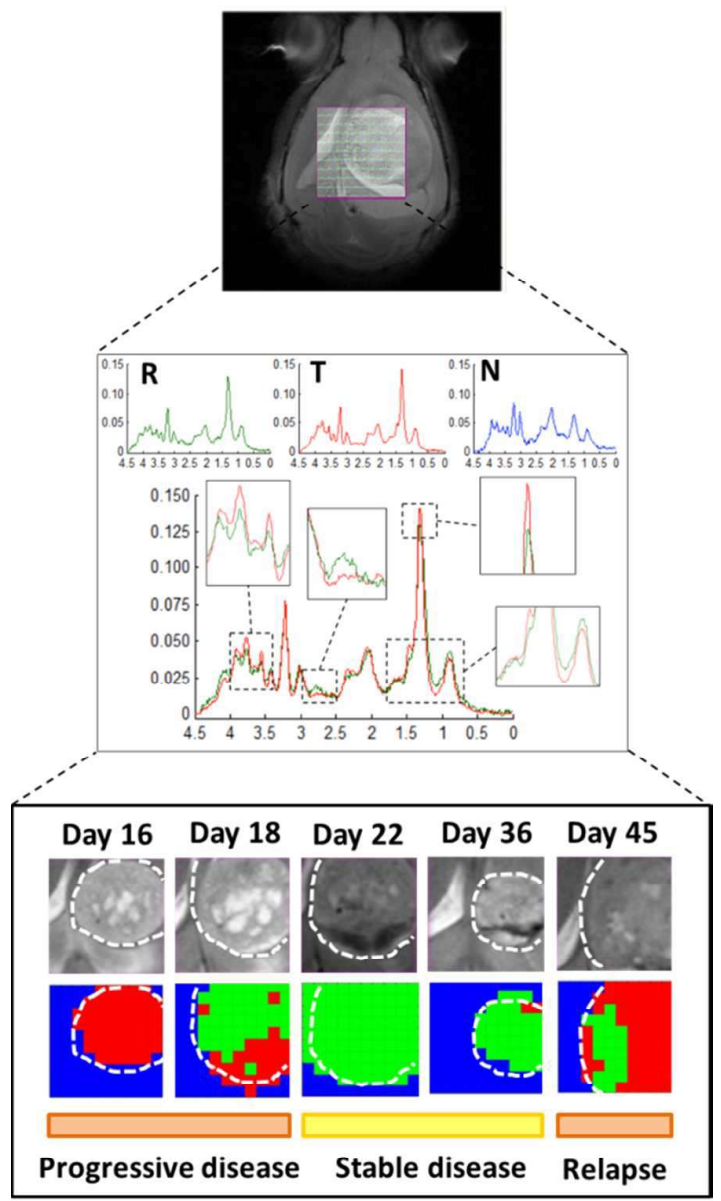



TEMOZOLOMIDE IN PRECLINICAL GLIOBLASTOMA USING SOURCE ANALYSIS

\section{AUTHORS AND AFFILIATIONS}

Delgado-Goñi $\mathrm{T}^{1,2, \dagger}$, Ortega-Martorell $\mathrm{S}^{3,2}$, Ciezka $\mathrm{M}^{1,2,5}$, Olier $\mathrm{I}^{4}$, Candiota $\mathrm{AP}^{2,1,5}$, Julià-Sapé $\mathrm{M}^{2,1,5}$, Fernández $\mathrm{F}^{6,2}$, Pumarola $\mathrm{M}^{6,2}$, Lisboa $\mathrm{PJ}^{3}$, Arús $\mathrm{C}^{1,2,5, \S}$.

1. Departament de Bioquímica i Biologia Molecular, Universitat Autònoma de Barcelona, Cerdanyola del Vallès, Spain.

2. Networking Research Center on Bioengineering, Biomaterials and Nanomedicine (CIBER-BBN), Universitat Autònoma de Barcelona, Cerdanyola del Vallès, Spain.

3. Department of Mathematics and Statistics, Liverpool John Moores University, Liverpool, United Kingdom.

4. Manchester Institute of Biotechnology, The University of Manchester, Manchester, United Kingdom.

5. Institut de Biotecnologia i de Biomedicina, Universitat Autònoma de Barcelona, Cerdanyola del Vallès, Spain.

6. Departament de Medicina i Cirurgia Animals, Universitat Autònoma de Barcelona, Cerdanyola del Vallès, Spain

${ }^{\dagger}$ Present address: Division of Radiotherapy and Imaging, The Institute of Cancer Research and The Royal Marsden NHS Foundation Trust, Sutton, Surrey, UK.

$\S$ Corresponding author: Carles Arús

Departament de Bioquímica i Biologia Molecular, Unitat de Bioquímica de Biociències, Edifici C, Universitat Autònoma de Barcelona, 08193 Cerdanyola del Vallès, Spain. Phone +34-93-581-1257; fax +34-93-581-1264. email carles.arus@uab.es

Running title: Glioblastoma therapy response detection by MRSI and source analysis.

WORD COUNT: 8146 (5687 not including references and captions).

KEY WORDS: Glioblastoma, Response detection, Magnetic Resonance Spectroscopic Imaging, Sources methodology, temozolomide. 


\begin{abstract}
ABREVIATIONS: DMSO, dimethyl sulfoxide; GB, glioblastoma; MDS, Multidimensional Scaling; MR, magnetic resonance; MRI, magnetic resonance imaging; MRS, magnetic resonance spectroscopy; MRSI, magnetic resonance spectroscopic imaging; NMF, Non-negative matrix factorization ; PE-MRSI, perturbation enhanced-MRSI; p.i., post-inoculation; PRESS, point resolved spectroscopy; RARE, Rapid Acquisition with Relaxation Enhancement; s.c., subcutaneous; SM, supplementary material; TE, echo time; TMZ, temozolomide; TR, repetition time; UL2, unit length; VOI, volume of interest.
\end{abstract}




\section{ABSTRACT}

\section{Purpose}

Characterization of Glioblastoma (GB) response to treatment is a key factor for improving patient's survival and prognosis. Magnetic Resonance Imaging and Spectroscopic Imaging (MRI/MRSI) provide morphologic and metabolic profiles of GB but usually fail to produce unequivocal biomarkers of response. The purpose of this work is to provide proof-of-concept of the capability of a semi-supervised signal source extraction methodology to produce images with robust recognition of response to temozolomide (TMZ) in a preclinical GB model.

\section{Materials and Methods}

A total of $38 \mathrm{C} 57 \mathrm{BL} / 6$ female mice were used in this study. The semi-supervised methodology extracted the required sources from a training set consisting of MRSI grids from 8 GL261 GB treated with TMZ, and 6 control untreated GB. Three different sources (normal brain parenchyma, actively proliferating GB and GB responding to treatment) were extracted and used for calculating nosologic maps representing the spatial response to treatment. These results were validated with an independent test set (7 control and 17 treated cases) and correlated with histopathology.

\section{Results}

Major differences between the responder and non-responder sources were mainly related to the resonances of mobile lipids (ML) and polyunsaturated fatty acids (PUFA) in ML (0.9, 1.3 and 2.8 ppm). Responding tumors showed significantly lower mitotic (3.3 \pm 2.9 vs. $14.1 \pm 4.2$ mitoses/field $)$ and proliferation rates $(29.8 \pm 10.3$ vs. $57.8 \pm 5.4 \%)$ than control untreated cases. 


\section{Conclusion}

The methodology described in this work is able to produce nosological images of response to TMZ in GL261 preclinical GB and suitably correlates with the histopathological analysis of tumors. A similar strategy could be devised for monitoring response to treatment in patients.

\section{INTRODUCTION}

Glioblastoma (GB) is the most common aggressive primary brain tumor type in adults (1), with a related survival rate of approximately 12-15 months with standard treatment (surgery followed by adjuvant radiotherapy and chemotherapy, e.g. Temozolomide, TMZ) (2). Throughout the period following standard therapy, the heterogeneity in its spatial distribution and growth dynamics, combined with interpatient variability, hamper personalized planning of further therapies (3).

Magnetic Resonance Imaging (MRI) and DCE-MRI are the standards for diagnosis and followup in GB patients $(1,4)$. Other methods for monitoring response to treatment are MRI-based ADC measurements, limited by the appearance of necrosis (5), and the emerging hyperpolarized ${ }^{13} \mathrm{C}$ magnetic resonance spectroscopy, that will be potentially translated to clinical cases (6). Magnetic Resonance Spectroscopy (MRS) and Spectroscopic Imaging (MRSI) are also used for monitoring the molecular properties and metabolic heterogeneity of brain tumors $(7,8)$. The rich information contained in MRS/MRSI signals makes them ideally suited to the application of pattern recognition (PR) techniques. However, for brain tumors and, more specifically, glial tumors, the computer-based discrimination of the grade or the tissue type is hampered by a "grey zone" of uncertainty between class labels (9). In this respect, signal source extraction applied to MRSI data constitutes a promising approach for unraveling characteristic spectral patterns of 
different conditions (tumor types, tissue types). A purely unsupervised approach is of interest when dealing with heterogeneous classes (e.g. glial tumors), despite the fact that prior information is then discarded (e.g. the diagnosis, or whether a tumor has received treatment or not). In (10), the authors proposed a semi-supervised methodology applied to SV MRS at 1.5T to guide signal source extraction towards the class labels of human brain tumor classes.

We have now applied such methodology to MRSI acquired at 7T to guide source extraction according to pre-existing information about TMZ therapy in the representative GL261 GB mouse model. We represented the spatial distribution of the sources as nosological images (11), i.e. colorcoded maps with normal brain tissue, responding tumor and non-responding tumor, and we validated the model retrospectively and prospectively with two groups of animals, one that had not received treatment and the second one, in which we obtained nosologic maps of the metabolomics of the tumor and surrounding tissue at two-day intervals during treatment and follow-up.

\section{MATERIALS AND METHODS}

\section{Ethics statement}

The use of animals in this study has been reported according to the ARRIVE guidelines (12).

Mice were obtained from Charles River Laboratories (France) and housed at the animal facility of the Universitat Autònoma de Barcelona (Servei d'Estabulari) https://estabulari.uab.cat. The husbandry conditions were as follows according to : Light/dark cycles: 12:12 h (light from 8 am to $8 \mathrm{pm})$; temperature of $20^{\circ} \mathrm{C}\left(+/-2^{\circ}\right)$; humidity of $55 \% \mathrm{Hr}(+/-10 \%)$; water and food access ad libitum (2014 Teklad Global 14\% Protein Rodent Maintenance Diet, Harlan, France); individually ventilated cages of 5 animals maximum (Tecniplast, Italy). The animal welfare was assessed weekly following a supervision protocol for brain tumor-bearing animals, in order to evaluate the 
severity of the symptoms generated by the growing masses. All studies were approved by the local ethics committee (Comissió d'Ètica en l'Experimentació Animal i Humana (CEEAH). Available: http://www.recerca.uab.es/ceeah. (Accessed 18 September 2015), according to the regional and state legislation (protocol DMAH-4600/CEEAH-1176/CEEAH-1391).

\section{Animals and Cells}

GL261 mouse glioma cells were obtained from the Tumor Bank Repository at the National Cancer Institute (Frederick/MD, USA) and were grown as previously described.

A total of $38 \mathrm{C} 57 \mathrm{BL} / 6$ female mice of $18-21$ weeks of age and 20-23g in weight, were included in this study. The weight of a representative group of control animals at the end of the study was $20.3 \pm 2.2 \mathrm{~g}(\mathrm{n}=11)$ and the weight of a representative group of treated animals was $19.7 \pm 2.9 \mathrm{~g}$ $(n=17)$.

\section{GL261 tumor generation}

Tumors were induced in the $38 \mathrm{C} 57 \mathrm{BL} / 6$ mice by an intracranial stereotactic injection of $10^{5}$ GL261 cells in the caudate nucleus (13).

The tumor-bearing animals were divided into a training group, consisting of 8 treated animals and 6 untreated controls, and two validation groups (see Table 1 for further details).

The first validation group was formed by 7 retrospective untreated control GL261 mice (14). The second validation group consisted of $17 \mathrm{TMZ}$ treated mice. In this second case, some treated tumors that were not supposed to respond to therapy (tumors treated only with one TMZ cycle, as reported in (15), $(\mathrm{n}=10))$ and one tumor treated with $3 \mathrm{TMZ}$ cycles were retrospectively included in order to assess the ability of the classifier to properly distinguish them. The remaining monitored animals $(n=6)$ were prospectively monitored every 2 to 3 days along the therapy protocol, in order to demonstrate the capability to track response and/or regrowth. 
The primary outcome (endpoint biomarker) considered in this study to establish antitumor activity for TMZ in the GL261 GB model was the overall survival time after tumor induction as described in (15). Furthermore, we also used other intermediate end point biomarkers as surrogates for primary outcome (16), such as tumor volume changes (in the longitudinal validation set) and Ki67 immunostaining (see sections on MRI studies and histopathology, respectively) in case animals were sacrificed before the endpoint (e.g. to assess proliferation by Ki67). The primary outcome and surrogate biomarkers were used to validate nosological images as additional non-invasive early biomarkers of response. For this the major metabolic changes induced in GL261 tumors by TMZ were assessed using MRSI to explore the animals during or after treatment administration, in order to characterize the tumour spectroscopic profile at specific timepoints. The results obtained were evaluated with the sources methodology (see below), providing a signature of response that enabled the recognition of responding and non-responding tumors.

All animals included in the training set $(n=14)$ were contemporary and were randomized after tumor volume calculation in the first MRSI acquisition (the volume at the beginning of the treatment was not significantly different from the value reported in (15) at day 10-12 postinoculation $\left.\left(16.9 \pm 6.2 \mathrm{~mm}^{3}\right)\right)$. After volume assessment animals were alternated between the control or treatment groups (17). The training set mice had previously been included in (15). With respect to the animals used in order to validate the therapy-response detection strategy, they were not contemporary to the training set. Thus, the seven GL261 controls had been analyzed in (14) and they were not randomized as all came from an experiment containing no treated animals. Furthermore, the 17 treated animals were prospectively generated and analyzed for the current study, also they were not randomized as all mice in this new set were treated. Still tumor volumes at day 10-12 post-inoculation were comparable to the training set cases. 


\section{TMZ administration}

TMZ was purchased from Sigma-Aldrich (Madrid, Spain), diluted in DMSO (AppliChem, Barcelona, Spain) $(200 \mathrm{mg} / \mathrm{ml})$ and sonicated: 4 pulses of $5 \mathrm{~s}$ at $30 \%$ of amplitude, interleaved with rest periods of 5 s (Ultrasonic Processor VCX750, Sonics \& Materials, Inc., Newtown, CT 06470-1614 USA).

Twenty-five animals were treated with TMZ following the three-cycle protocol described at (15). Each animal received a dose of $60 \mathrm{mg} / \mathrm{Kg}$ per day of treatment. The volume administered with an intragastric probe $(20 \mathrm{G}, 38 \mathrm{~mm}$, Popper \& Sons, New York, USA) was $10 \mu 1$ per gram of weight.

\section{In vivo MRI and MRSI studies}

MR studies were carried out at the joint NMR facility of UAB and CIBER-BBN, Unit 25 of NANBIOSIS, with a 7T horizontal magnet (BioSpec 70/30, Bruker BioSpin, Ettlingen, Germany) equipped with actively shielded gradients (B-GA12 gradient coil inserted into a B-GA20S gradient system) and a quadrature receive surface coil, actively decoupled from a volume resonator with $72 \mathrm{~mm}$ inner diameter

\section{MRI studies}

The tumor-bearing mice were explored by MRI for measuring tumor volume and calculating their respective growth curve profiles the same day when the MRSI sequence was acquired (see Supplementary Material). In the case of the longitudinal study with 6 animals of the test set, tumors were scanned every 2 to 3 days after the initial exploration day (day 10-12 post inoculation). Tumor volume response to treatment (intermediate endpoint biomarker) was evaluated according to the RECIST criteria (18): partial response is defined by at least a $30 \%$ decrease in the sum of diameters of target lesions, taking as reference the baseline sum diameters 
(in this case the volume in the previous exploration); progressive disease involves at least a $20 \%$ increase in the volume of target lesions, taking as reference the smallest volume calculated in the previous exploration, and stable disease is defined by neither sufficient shrinkage to qualify for partial response nor sufficient increase to qualify for progressive disease, taking as reference the smallest volume while on study.

\section{MRSI studies}

All animals studied in this work were scanned by MRSI, at different time points of the therapy protocol and most were sacrificed afterwards in order to correlate the metabolic spectral pattern of the tumors with their corresponding histopathology analysis. In the case of the 6 animals from the prospective validation group included in the longitudinal study, tumors were monitored by MRSI every 2-3 days along the therapy protocol for a better and more complete characterization of the response along time and to gather primary outcome values (overall survival). The histopathological samples were collected the last day of the protocol after the corresponding MRSI study.

MRI and MRSI sequences used in this work are described in the SM section.

All the animals were studied by MRSI at different time-points during the TMZ treatment protocol as in (15). Control animals were explored once between days 10 and 18 post-inoculation and treated animals were explored a minimum of one (training group) and a maximum of 18 times (validation group) between days 15 and 45 post-inoculation at 2-3 day intervals. MRSI grids were spatially located to allow the volume of interest (VOI) to include most of the tumoral mass as well as normal/peritumoral brain parenchyma. 


\section{MR Processing}

MRI T2w high-resolution images were used for tumor volume calculation (See SM). MRSI data were post-processed essentially as described in (19) and (20). Briefly, data were initially preprocessed at the MR workstation with ParaVision 5.0 (Bruker BioSpin), and later post-processed with 3DiCSI v1.9.10 (21) and exported in ASCII format. Dynamic MRSI processing Module (DMPM) (22) running over MatLab (The MathWorks Inc., Natick, MA, USA), was used to align and normalize all the spectra to UL2 (13).

\section{Voxel labeling and Signal source extraction}

Non-negative Matrix Factorization (NMF) methods belong to a group of multivariate data analysis techniques aimed to estimate meaningful latent components, also known as sources, from non-negative data. Standard NMF methods decompose the data matrix X into two non-negative matrices $\mathrm{S}$ (the sources) and A (the mixing matrix). The differences between those analysis techniques are given by the different cost functions used for measuring the divergence between $\mathrm{X}$ and S*A. In (23) the authors presented a variant of NMF, namely Convex-NMF, in which the basis vectors of $\mathrm{S}$ are constrained to be convex combinations of the data points. Convex-NMF relaxes the NMF constraints to allow negative values both in the data matrix and the sources. The semi-supervised methodology described in (10) was applied for the extraction of meaningful source signals in the tumors from the training set, regarding the spectral vectors selected from the original MRSI grids, (see SM for further details and Figure S1).

\section{Nosological imaging of the response to therapy}

Equation (1) (were $F$ is the source matrix, $H$ is the mixing matrix, $X$ is the original data matrix, $T$ represents the transpose, $(\cdot)^{+}$is the positive part of the matrix, where all negative values become zero, and $(\cdot)^{-}$is the negative part of the matrix, where all positive values become zero) fixed the 
values for the extracted sources and was applied for calculating the mixing matrices $(\mathrm{H})$ of each individual case, and with them, the nosological images representing the proportions in which each source is being represented in each voxel (10).

$$
H_{n k} \leftarrow H_{n k} \sqrt{\frac{\left(X^{T} F\right)_{n k}^{+}+\left[H\left(F^{T} F\right)^{-}\right]_{n k}}{\left(X^{T} F\right)_{n k}^{-}+\left[H\left(F^{T} F\right)^{+}\right]_{n k}}}
$$

A total of 508 spectral vectors from the training set $(n=14$ mice, table 1) were included in the analysis. From the 100 voxels included in the MRSI VOI of each animal, those located in the edges of the grid, with a signal to noise ratio (SNR, calculated as in (24)) lower than $57.2 \pm 26.0$ were discarded; and those located in the tumor boundaries with normal tissue were also discarded to avoid spectral pattern mixing or contamination from different tissue types essentially as described in $(14,25)$. Voxels outside the tumoral mass (as shown by T2w MRI), showing a high accumulation of DMSO in the color-coded maps calculated from PE-MRSI sequences (see (19), were also discarded for training purposes: we have previously reported that DMSO accumulates preferentially in tumoral tissue with respect to the surrounding normal brain parenchyma (19). Nonetheless, there are DMSO retention hot-spots, external to the major T2w hyperintensity tumor mass, which origin is still unclear. For extracting the main sources in the present work we decided to include only "pure" voxels of known nature (from the MRI/MRSI image perspective) that could help us to maximize the differences detectable between untreated tumors and tumors responding to TMZ therapy. The selected spectral vectors were labeled as tumor (responding and control) and normal parenchyma, initially assuming that all the voxels inside each tumor belonged to the same category (responding or control). The robustness of this assumption was later on evaluated using both the training set and the independent test set described in Methods.

The $80 \%$ of those 508 spectral vectors (randomly selected) were used to create the Multi-Layer Perceptron (MLP) model, which was assessed with the remaining $20 \%$ of them. This model was used to estimate the conditional probabilities of class membership for each case, which were then 
used to define the Fisher Information metric. Three sources were calculated afterwards, following the three steps of the semi-supervised methodology mentioned before, one of them to represent the responding tumor, another to represent the tumor without treatment, and a third for the normal tissue.

As we labeled each voxel by selecting the source with highest correlation with it, we needed to test whether there were significant differences between choosing this particular label or another. Therefore, for each case in the training set, we compared them by using a Student's t-test and confirmed a $p$-value $p<0.05$, which indicated that the differences are significant in every case. If the correlation between the spectrum of a voxel and each of the sources was below a threshold of $50 \%$, then we labeled this voxel as 'undecided' (thus effectively abstaining from labeling the voxel) and were colored in black.

\section{Histopathology}

After the last MRSI study, 32 animals (10 controls and 22 treated) were sacrificed with an i.p. injection of pentobarbital $(200 \mathrm{mg} / \mathrm{kg}, 60 \mathrm{mg} / \mathrm{mL})$. Brains were immediately collected, fixed in $4 \%$ formaldehyde and analyzed by histopathology as described in (15). Hematoxylin and Eosin (HE) staining, performed in coronal brain slices at different heights, was used for correlating the histological slices with the reference image in the MRSI studies and for a microscopic evaluation of the presence of necrosis. Caspase 3 (Cell Signalling Technology, Izasa, Barcelona) and Ki67 (BD Biosciencies, Madrid) immunohistochemical stainings were used for detecting apoptosis and cell proliferation (intermediate endpoint biomarker), respectively, in treated and untreated tumors. The histopathological slides were digitized for further quantification (Hamamatsu Photonics France SARL, Massy, France). 


\section{Statistical analysis.}

Part of the statistical analysis was carried out with the SPSS 20.0 (IBM SPSS Statistics, U.S.) and G-Stat 2.0 software (GlaxoSmithKline S.A., Madrid). Tumor volume differences between groups along the therapy protocol were calculated using the Mann-Whitney U test for independent samples and Student's t-test, depending on sample normality. The ANOVA test applying Bonferroni correction was used for comparing the immunohistochemical quantifications for Ki67, Caspase 3 and mitoses between control and treated groups. The presence of outliers in Caspase 3 samples was tested with Grubb's and Dixon's tests. The extent to which the semisupervised methodology provided the right labeling to each group or class was assessed by a Student's t-test, from the stats package in R. The differences between histopathology data from each case and the global threshold defined in (15) were assessed using the one sample Student's ttest. A contingency analysis based on Chi-Square tests and Phi and Cramer's V symmetric measures, determined the correlation between the primary outcome (overall survival), intermediate endpoint biomarkers (RECIST response, proliferation (Ki67) and apoptosis (Caspase 3)) and necrosis with the response detected by the sources methodology. Student's t-test was applied to compare Ki67 staining between responding and not responding tumors. Significance was accepted at $\mathrm{p}<0.05$ unless stated otherwise.

\section{RESULTS}

Semi-supervised source extraction in the training set and corresponding nosologic maps at single growth curve time points

The growth curves for GL261 tumors in the training and test sets are shown in SM (Figure S2). Figure 1 illustrates the source signals obtained using the semi-supervised methodology within the 
training set, which represent the three tissue types studied: normal brain parenchyma (N), GL261 tumors treated with $3 \mathrm{TMZ}$ cycles (mostly in response or as stable disease) (R) and untreated control GL261 tumors (actively proliferating) (T).

The nature of the changes in the spectral pattern of treated tumors is mainly related to mobile lipids (0.9 and $1.3 \mathrm{ppm}$ ) and polyunsaturated fatty acids in mobile lipids (PUFA) (2.8 ppm) resonances. Still, other contributions (e.g. lactate (1.3 and $4.1 \mathrm{ppm})$, glutamine, glutamate, alanine (3.8 ppm), and myo-inositol/glycine (3.5 ppm)) (see Figure 1) may be helping discrimination between tumor sources.

As explained in Material and Methods, the correlations between the sources and the MR spectrum of each voxel in the MRSI grids were used for calculating nosologic images. Figure 2A illustrates the three main groups of nosologic patterns detected by the source methodology in the training set of this work for the region recognized as tumor: tumors with a clear control proliferating pattern (C583), tumors clearly responding to treatment (C586) and treated or control tumors with a partial or heterogeneous pattern of response (C418). The sensitivity/specificity values calculated for the sources related to tumor $(\mathrm{T}+\mathrm{R})$ and non-tumor $(\mathrm{N})$ (see table $\mathrm{S} 1$ in $\mathrm{SM}$ ) are comparable to those described in using two sources (between 1-0.97 and 1-0.86 respectively).

The eleven remaining cases from the training group are shown in Figure 3. In short, 4 out of the 6 control mice from the training group were recognized mostly as T, while two of them $(\mathrm{C} 351$, C529) had 48.3 and $35.8 \%$ of spectral vectors recognized as R. For the treated mice, only 2 out of 8 were recognized mostly as R, in 3 out of 8 there was regional heterogeneity of response and in 3 out of 8 the tumor tissue was recognized as T (control proliferating pattern). These suboptimal classification results within the training group could be due to an inadequate representation of the tissue type associated to the class prototype for tumor tissue, or to the intrinsic heterogeneity of both control and treated tumors. 
In this respect, histopathological analysis (Table S2) could be performed in 10 of the animals in the training set ( 3 controls and 7 treated). Each individual training case was also compared with the average values for control tumors and those cases treated with TMZ described in (15) (Figure 2B). Several training group cases were included within the box plot range described for their reference group (control or treated) and were correctly recognized by the sources methodology.

Regarding the tumors with mixed pattern ( $\mathrm{T}$ and R), four treated cases (C415, C418, C525 and C527) showed a significantly higher proliferation rate than the treated group average described in (15) $(\mathrm{p}=0.001$, Table $\mathrm{S} 2$ and Figure $2 \mathrm{~B})$, and were accordingly recognized as full/partial nonresponders in the nosologic maps (Figure 2A and 3). No histopathology data was available for case C351. Further details on the histopathology of individual cases are provided in SM.

In summary the source-based classifier developed with the training set finds regions of heterogeneity compatible with restricted growth in control tumors, while the average response pattern found for TMZ treated tumors in (15) is heterogeneous across tumors and even within individual tumors. To further evaluate the robustness of the developed classifier, an additional study on an independent test set of tumors was performed.

Source validation by two independent groups of animals. Individual time points and longitudinal studies.

To test the validity of the proposed methodology, independent cases (control and treated) were analyzed using the sources extracted from the training set to generate new mixing matrices. Nosologic maps were produced for each case, and these images were correlated and validated with the corresponding histopathological studies, and RECIST criteria of response (see SM for details) in the longitudinal study group. 
Based on the nosologic images generated (Figure 4), these two independent groups of cases could be subdivided into three categories: One group of untreated tumors that showed clear progression (the 7 retrospective control cases shown in Figure 4). This classification agreed well with their proliferation index (Table S3 and (14)), and with average values for reference GL261 tumors in (15). A second group of treated tumors with no apparent response to TMZ (animals treated with only one TMZ cycle with nosologic maps displayed in Figure S3 of SM and histopathology data shown in Table S3). And a third group of treated tumors (the prospective group) responding to treatment (Figure 4 and Table S3).

Regarding the prospective group, 6 of the mice were included in a longitudinal study. Nosologic maps calculated the day just prior to death/ethical sacrifice are shown in Figure 4, when tumor proliferation assessed by histopathology (Table S3) was significantly arrested, according to the box plot range for tumors treated with $3 \mathrm{TMZ}$ cycles (Figure 2B). The response throughout the course of therapy for all of them is shown in Figure 5. Tumors' response to treatment started to be detectable between days 15 and 23, before changes in tumor volume with respect to average values of control untreated tumors (Table S3 and (14)) were observed (see Figure S2). For the longitudinal follow up of these treated mice, it may be more informative to consider the correlation between the time frame when tumor spectral vectors were recognized as responding, and the RECIST evaluation of the individual time points. Then, for example, tumor volume for case C819 indicates it entered the stable disease state at day 22 (neither $30 \%$ of volume reduction nor $20 \%$ of volume increase with respect to the previous measurement) in agreement with fully responding spectral vectors in the nosological image, and remained in a transient response state until day 26 , when it showed partial response $(30 \%$ of volume reduction with respect to the previous measurement). Then it remained stable according to RECIST until day 41 (when it showed more than $20 \%$ of volume increase with respect to day 39 ), which agrees with mostly responding spectral vectors in the tumor mass during this period, and an active proliferation 
pattern at day 45. Stable disease periods were also found for mice C776, C808 and C821 (Figure S2C), which also agreed for responding nosological images in figure 5. The only apparently "abnormal" case is C795, discussed in SM.

Finally, a global analysis was performed including all cases from the training and validation groups that had histopathology data available and with apparently homogeneous nosologic maps (more than $50 \%$ of the pixels responding or not responding), in order to detect if there was correlation between both types of response biomarkers. The corresponding contingency tables are included in Figure 6A and S4. The primary outcome (overall survival), was considered in the 9 animals that were treated with $3 \mathrm{TMZ}$ cycles (the rest were sacrificed before protocol completion to correlate the sources methodology with the well-known surrogate endpoint biomarker, tumor proliferation assessed by Ki67). The survival of these animals was comparable to that reported in (15) for GL261 tumor-bearing mice treated with 3 TMZ cycles (Figure S4) and was significantly correlated with the response detected by the sources methodology (Phi $=0.89, \mathrm{p}<0.001$, Figure S4). Moreover, the RECIST response (considering response either stable disease or partial response) for each case in the prospective group of the validation set $(n=6)$ at the days of the MRSI exploration was also significantly correlated with the corresponding nosologic maps (Phi $=$ $0.73, \mathrm{p}<0.001$, Figure S4). The Ki67 proliferation index showed a strong correlation with the response detected using the sources methodology $(\mathrm{Phi}=-0.85, \mathrm{p}<0.001)$, indicating that higher proliferation rates correlate with lack of response in the nosologic maps (Figure 6A). No relationship was detected between Caspase 3 (Figure 6A) or microscopic necrosis (based on Hematoxilin-eosin staining, Figure S4) values and the sources results. A further analysis comparing the Ki67 indexes of cases with not responding nosologic maps with those showing responding maps demonstrated a significantly lower proliferation rate in responding-source cases (Figure 6B). 


\section{DISCUSSION}

MRSI and semi-supervised source extraction methodology as a non-invasive approach for GB response to treatment characterization

We have used a non-invasive methodology (MRSI-based nosological images) that allows us to distinguish when a GL261 GB tumor is responding (totally or partially) to the TMZ therapy, in both, training and test cases. That is: 1) the treated cases where there was indication of therapy response were mainly identified as responding; 2) the treated cases where there were signs of regrowth/relapse of the tumor were mainly identified as untreated/not responding; and 3) the control cases were mainly identified as untreated/not responding. As indicators of response to therapy we used overall survival (primary outcome) and the volume of the tumor mass (RECIST criteria) where possible (longitudinal cases) or the histopathology (Ki67 labelling), as intermediate endpoint biomarkers, which demonstrated to be strongly correlated with the source-based nosologic maps (Figures 6 and S4).

Strikingly, the methodology was able to see beyond the original labels used in the first part of the training process (schematized in Figure S1), where we grouped together in one class all the tumor spectra from the treated cases (regardless of their level of response to therapy), in another class all the tumor spectra from the control cases, and in a third class all the normal parenchyma spectra. The methodology clearly separated the normal brain, and found the subtle differences between the two tumor sources that are able to differentiate response from untreated/not responding. This demonstrates the learning ability of this methodology, which managed to make a good generalization of the problem at hand and allowed us to build a robust model for the analysis not only of the data used for training but also the test (out-of-sample) cases.

The sources extracted using the semi-supervised methodology are visually similar to the average spectra of normal brain parenchyma and GB already described in previous work (10). 
Moreover, the major differences between the three sources (normal tissue, control tumor and tumor responding to treatment), also agree with previous work. Thus, higher mobile lipids, low NAA and a higher choline / creatine ratio differentiate control tumor spectra from normal brain parenchyma (20). Mobile lipids and polyunsaturated fatty acids (PUFA) signals in mobile lipids are higher in responsive tumors in comparison to untreated/unresponsive tumors, indicating therapy-related metabolic changes, mostly based on apoptosis induced by treatment $(26,27)$. Other minor differences among sources (e.g. in the lactate, alanine and myo-inositol/glycine spectral regions, see Figure 1) may be helping the differentiation between response and no response (28). For further experiments it could be interesting to test whether long echo time (135-144 ms) based sources allow a similar discrimination of responding and non-responding tumors, helping to better characterise the potential contribution of lactate at $1.3 \mathrm{ppm}$ to the classification (29).

Some of the cases used in the training set showed a clearly mixed pattern between responding and non-responding regions. However, as mentioned above, this did not compromise the source extraction process. The segmentation of different tissue types (responding/not responding) within the same tumor would agree with an intrinsic intratumoral heterogeneity being unveiled in vivo by the source analysis. The intratumoral heterogeneity in individual cases should be related to the intrinsic heterogeneity of GB which is nowadays well recognized (30).

The performance of the presently proposed method for therapy response recognition was compared for a selected number of cases (see SM results) to the previously developed method based in supervised classifier development (15) and found better performing (see SM). 


\section{Correlation between calculated nosologic maps and histopathology}

A significant correlation was obtained comparing the nosologic maps with the proliferation stage of the tumors (higher proliferation meaning lack of response) (Figure 6). Even cases that were supposed to be responding (C415 and C525) or not responding (C419), according to the type of treatment received, were correctly recognized by the sources as non responders ( $\mathrm{C} 415$ and C525) and responders (C419), in agreement with their respective proliferation indexes. In this respect, Ki67 staining has been reported as a good response biomarker for preclinical (31) and clinical GB, also related to patient survival and time to recurrence $(32,33)$, and, in some cases, this parameter seems to be more effective for response evaluation than the RECIST criteria (34). Our proposed methodology provides an alternative non-invasive analysis with comparable efficacy to both the primary outcome and intermediate surrogate end-point biomarkers accepted in the clinic, demonstrating translational potential. In this respect, the here described use of DMSO to discard voxels outside the abnormal mass in the initial source extraction process may not be mandatory in the case of patients, although further work will be required to clarify this.

The response maps calculated with the sources methodology did not correlate well with caspase 3 staining or necrosis quantification performed both in the training and test set cases. In the first case, this could be due to the fact that untreated GB have been reported to inherently express activated caspase 3 as part of their intrinsic process of cell death $(35,36)$ or even related to migration and invasiveness (37). The responding source profile shows an increase in PUFAs, which seem to be related to cumulative apoptosis (38), whereas caspase 3 content correlates with early stage apoptosis (39), indicating that a different staining, such as TUNEL (40), may be needed to better correlate cumulative apoptosis with the changes detected in the nosologic maps. In the case of necrosis, the lack of correlation could be explained by the fact that there were no differences in the number of not responding tumors between low and high necrosis groups. These 
results suggest that control tumors growing exponentially are more susceptible to develop necrosis (41), which is one of the markers for poor prognosis in the clinic $(42,43)$ but this is a heterogeneous process linked to tumor progression stage (44) which in our case does not correlate with response or the lack of it.

\section{Translational potential and limitations of the proposed study.}

One of the main challenges regarding brain tumor diagnoses and follow up during treatment in the clinic, is to select an accurate non-invasive technique able to provide detailed metabolic information about the whole tumoral mass. The sources methodology described in this work demonstrates a significant potential for tumor longitudinal response characterization, able to distinguish heterogeneous areas inside the tumors. Although it still shows some limitations, related to the characterization of some regions (classified as undefined tissue), or to the discrimination between normal parenchyma and tumor progression in some cases, these could be implemented in the future to provide an even more accurate response characterization. One of the major changes detected by the source methodology, that enables the differentiation between response and progression, involves resonances in mobile lipids and polyunsaturated fatty acids (PUFA) in mobile lipids. These have been reported as brain tumor response biomarkers indicating therapyinduced apoptosis (26), which can be induced in brain tumors by different treatments (45-47) including radiotherapy, commonly administered in combination with TMZ in the clinic, but not used in the present work. Nonetheless, therapies based on different mechanisms (e.g antiangiogenic agents (29) may induce different metabolic changes compared to classical chemotherapeutic agents. Accordingly, the robustness of the therapy detection classifiers may need to be revaluated for the different agents used and, in case of need, adequate retraining performed to ascertain and use their pattern specificities for response discrimination. One 
additional limitation to consider for translational work is that the use of DMSO for PE-MRSI in patients is not yet authorized by regulatory agencies. Accordingly, either such clearance is obtained first or training ignoring possible retention hot-spots outside the tumor mass may be initially attempted for human use.

Finally, another aspect to take into account with respect to translational potential in clinical cases, is the location of the tumor. This, if close to the scalp, may affect data quality and an appropriate fat suppression sequence should be applied to obtain acceptable results. Nowadays different acquisition protocols are being investigated to improve fat suppression in MRSI studies in the clinic $(48,49)$, so this issue should not compromise the translational potential of our method.

\section{CONCLUSION}

The methodology described in this work is able to characterize the response to TMZ in preclinical GL261 GB both at a defined time-point and in real time, along the therapy protocol. The response patterns detected correlate well with the histopathological analysis of control and treated tumors, at each stage. This strategy bears potential for monitoring tumor response to treatment and allowing early decision making with respect to second line therapeutic strategies both for preclinical models and translational application in patients .

\section{ACKNOWLEDGMENTS}

The authors would like to thank Dr Qi Zhao and Dr Radka Stoyanova for introducing the necessary modifications in 3DiCSI software to read our data and Dr Rui Simões for allowing the use of previously described data in (14). We also wish to thank Ester Blasco and Lola Pérez, from the veterinary neuropathology group at $U A B$, for technical assistance. Time allocation in the 
Servei de Ressonància Magnètica Nuclear at the Universitat Autònoma de Barcelona is gratefully acknowledged.

This work was funded by MINECO grants MARESCAN (SAF 2011-23870) and MOLIMAGLIO (SAF2014-52332-R), and by Centro de Investigación Biomédica en Red-Bioingeniería, Biomateriales y Nanomedicina (CIBER-BBN, [http://www.ciber-bbn.es/en]), an initiative of the Instituto de Salud Carlos III (Spain) co-funded by EU FEDER. M. Ciezka held a FI-DGR grant from the Generalitat de Catalunya. S. Ortega-Martorell is funded by the European Union under the 7th FP, "Marie Curie" FP7-PEOPLE-2012-IEF.

Results shown in this work were partially communicated in two oral presentations $(50,51)$.

\section{REFERENCES}

1. Brandes AA, Tosoni A, Franceschi E, Reni M, Gatta G, Vecht C. Glioblastoma in adults. Crit Rev Oncol Hematol 2008;67(2):139-152.

2. Rhee DJ, Kong DS, Kim WS, Park KB, Lee JI, Suh YL, Song SY, Kim ST, Lim DH, Park $\mathrm{K}$, Kim JH, Nam DH. Efficacy of temozolomide as adjuvant chemotherapy after postsurgical radiotherapy alone for glioblastomas. Clin Neurol Neurosurg 2009;111(9):748-751.

3. Neal ML, Trister AD, Ahn S, Baldock A, Bridge CA, Guyman L, Lange J, Sodt R, Cloke T, Lai A, Cloughesy TF, Mrugala MM, Rockhill JK, Rockne RC, Swanson KR. Response classification based on a minimal model of glioblastoma growth is prognostic for clinical outcomes and distinguishes progression from pseudoprogression. Cancer Res 2013;73(10):2976-2986.

4. Suh CH, Kim HS, Choi YJ, Kim N, Kim SJ. Prediction of pseudoprogression in patients with glioblastomas using the initial and final area under the curves ratio derived from dynamic contrast-enhanced T1-weighted perfusion MR imaging. AJNR Am J Neuroradiol 2013;34(12):2278-2286.

5. Debnam JM, Schellingerhout D. Diffusion MR Imaging of the Brain in Patients with Cancer. Int J Mol Imaging 2011:714021.

6. Kurhanewicz J, Vigneron DB, Brindle K, Chekmenev EY, Comment A, Cunningham CH, Deberardinis RJ, Green GG, Leach MO, Rajan SS, Rizi RR, Ross BD, Warren WS, Malloy CR. Analysis of cancer metabolism by imaging hyperpolarized nuclei: prospects for translation to clinical research. Neoplasia 2011;13(2):81-97.

7. Nelson SJ. Assessment of therapeutic response and treatment planning for brain tumors using metabolic and physiological MRI. NMR in biomedicine 2011;24(6):734-749.

8. Segebarth CM, Baleriaux DF, Luyten PR, den Hollander JA. Detection of metabolic heterogeneity of human intracranial tumors in vivo by $1 \mathrm{H}$ NMR spectroscopic imaging. Magn Reson Med 1990;13(1):62-76. 
9. Coons SW, Johnson PC, Scheithauer BW, Yates AJ, Pearl DK. Improving diagnostic accuracy and interobserver concordance in the classification and grading of primary gliomas. Cancer 1997;79(7):1381-1393.

10. Ortega-Martorell S, Ruiz H, Vellido A, Olier I, Romero E, Julia-Sape M, Martin JD, Jarman IH, Arus C, Lisboa PJ. A novel semi-supervised methodology for extracting tumor type-specific MRS sources in human brain data. PLoS One 2013;8(12):e83773.

11. De Edelenyi FS, Rubin C, Esteve F, Grand S, Decorps M, Lefournier V, Le Bas JF, Remy C. A new approach for analyzing proton magnetic resonance spectroscopic images of brain tumors: nosologic images. Nat Med 2000;6(11):1287-1289.

12. Kilkenny C, Browne WJ, Cuthill IC, Emerson M, Altman DG. Improving bioscience research reporting: the ARRIVE guidelines for reporting animal research. PLoS biology 2010;8(6):e1000412.

13. Simoes RV, Garcia-Martin ML, Cerdan S, Arus C. Perturbation of mouse glioma MRS pattern by induced acute hyperglycemia. NMR in biomedicine 2008;21(3):251-264.

14. Ortega-Martorell S, Lisboa PJ, Vellido A, Simoes RV, Pumarola M, Julia-Sape M, Arus C. Convex Non-Negative Matrix Factorization for Brain Tumor Delimitation from MRSI Data. PLoS One 2012;7(10):e47824.

15. Delgado-Goni T, Julia-Sape M, Candiota AP, Pumarola M, Arus C. Molecular imaging coupled to pattern recognition distinguishes response to temozolomide in preclinical glioblastoma. NMR in biomedicine 2014;27(11):1333-1345.

16. de Bono JS, Ashworth A. Translating cancer research into targeted therapeutics. Nature 2010;467(7315):543-549.

17. El Meskini R, Iacovelli AJ, Kulaga A, Gumprecht M, Martin PL, Baran M, Householder DB, Van Dyke T, Weaver Ohler Z. A preclinical orthotopic model for glioblastoma recapitulates key features of human tumors and demonstrates sensitivity to a combination of MEK and PI3K pathway inhibitors. Disease models \& mechanisms 2015;8(1):45-56.

18. Eisenhauer EA, Therasse P, Bogaerts J, Schwartz LH, Sargent D, Ford R, Dancey J, Arbuck S, Gwyther S, Mooney M, Rubinstein L, Shankar L, Dodd L, Kaplan R, Lacombe D, Verweij J. New response evaluation criteria in solid tumours: revised RECIST guideline (version 1.1). Eur J Cancer 2009;45(2):228-247.

19. Delgado-Goni T, Martin-Sitjar J, Simoes RV, Acosta M, Lope-Piedrafita S, Arus C. Dimethyl sulfoxide (DMSO) as a potential contrast agent for brain tumors. NMR in biomedicine 2013;26(2):173-184.

20. Simoes RV, Delgado-Goni T, Lope-Piedrafita S, Arus C. 1H-MRSI pattern perturbation in a mouse glioma: the effects of acute hyperglycemia and moderate hypothermia. NMR in biomedicine 2010;23(1):23-33.

21. http://mrs.cpmc.columbia.edu/3dicsi.html.

22. http://gabrmn.uab.es/DMPM.

23. Paatero P, Tepper U. Positive matrix factorization: A non-negative factor model with optimal utilization of error estimates of data values. . Environmetrics 1994;5(2):111-126.

24. Tate AR, Underwood J, Acosta DM, Julia-Sape M, Majos C, Moreno-Torres A, Howe FA, van der Graaf M, Lefournier V, Murphy MM, Loosemore A, Ladroue C, Wesseling P, Luc Bosson J, Cabanas ME, Simonetti AW, Gajewicz W, Calvar J, Capdevila A, Wilkins PR, Bell BA, Remy C, Heerschap A, Watson D, Griffiths JR, Arus C. Development of a decision support system for diagnosis and grading of brain tumours using in vivo magnetic resonance single voxel spectra. NMR in biomedicine 2006;19(4):411-434.

25. Simoes RV, Ortega-Martorell S, Delgado-Goni T, Le Fur Y, Pumarola M, Candiota AP, Martin J, Stoyanova R, Cozzone PJ, Julia-Sape M, Arus C. Improving the classification of brain tumors in mice with perturbation enhanced (PE)-MRSI. Integr Biol (Camb) 2012;4(2):183-191. 
26. Hakumaki JM, Poptani H, Sandmair AM, Yla-Herttuala S, Kauppinen RA. 1H MRS detects polyunsaturated fatty acid accumulation during gene therapy of glioma: implications for the in vivo detection of apoptosis. Nat Med 1999;5(11):1323-1327.

27. Mirbahai L, Wilson M, Shaw CS, McConville C, Malcomson RD, Kauppinen RA, Peet AC. Lipid biomarkers of glioma cell growth arrest and cell death detected by $1 \mathrm{H}$ magic angle spinning MRS. NMR in biomedicine 2012;25(11):1253-1262.

28. Wei L, Hong S, Yoon Y, Hwang SN, Park JC, Zhang Z, Olson JJ, Hu XP, Shim H. Early prediction of response to Vorinostat in an orthotopic rat glioma model. NMR Biomed 2012;25(9):1104-1111.

29. Hamans B, Navis AC, Wright A, Wesseling P, Heerschap A, Leenders W. Multivoxel (1)H MR spectroscopy is superior to contrast-enhanced MRI for response assessment after anti-angiogenic treatment of orthotopic human glioma xenografts and provides handles for metabolic targeting. Neuro-oncology 2013;15(12):1615-1624.

30. Sottoriva A, Spiteri I, Piccirillo SG, Touloumis A, Collins VP, Marioni JC, Curtis C, Watts $\mathrm{C}$, Tavare S. Intratumor heterogeneity in human glioblastoma reflects cancer evolutionary dynamics. Proceedings of the National Academy of Sciences of the United States of America 2013;110(10):4009-4014.

31. Marrero L, Wyczechowska D, Musto AE, Wilk A, Vashistha H, Zapata A, Walker C, Velasco-Gonzalez C, Parsons C, Wieland S, Levitt D, Reiss K, Prakash O. Therapeutic efficacy of aldoxorubicin in an intracranial xenograft mouse model of human glioblastoma. Neoplasia 2014;16(10):874-882.

32. Burton EC, Lamborn KR, Forsyth P, Scott J, O'Campo J, Uyehara-Lock J, Prados M, Berger M, Passe S, Uhm J, O'Neill BP, Jenkins RB, Aldape KD. Aberrant p53, mdm2, and proliferation differ in glioblastomas from long-term compared with typical survivors. Clinical cancer research : an official journal of the American Association for Cancer Research 2002;8(1):180-187.

33. Pouleau HB, Sadeghi N, Baleriaux D, Melot C, De Witte O, Lefranc F. High levels of cellular proliferation predict pseudoprogression in glioblastoma patients. International journal of oncology 2012;40(4):923-928.

34. Kurokawa Y, Shibata T, Ando N, Seki S, Mukaida H, Fukuda H. Which is the optimal response criteria for evaluating preoperative treatment in esophageal cancer: RECIST or histology? Ann Surg Oncol 2013;20(9):3009-3014.

35. Ray SK, Patel SJ, Welsh CT, Wilford GG, Hogan EL, Banik NL. Molecular evidence of apoptotic death in malignant brain tumors including glioblastoma multiforme: upregulation of calpain and caspase-3. Journal of neuroscience research 2002;69(2):197-206.

36. Saggioro FP, Neder L, Stavale JN, Paixao-Becker AN, Malheiros SM, Soares FA, Pittella JE, Matias CC, Colli BO, Carlotti CG, Jr., Franco M. Fas, FasL, and cleaved caspases 8 and 3 in glioblastomas: a tissue microarray-based study. Pathology, research and practice 2014;210(5):267-273.

37. Gdynia G, Grund K, Eckert A, Bock BC, Funke B, Macher-Goeppinger S, Sieber S, Herold-Mende C, Wiestler B, Wiestler OD, Roth W. Basal caspase activity promotes migration and invasiveness in glioblastoma cells. Molecular cancer research : MCR 2007;5(12):1232-1240.

38. Jimenez-Xarrie E, Davila M, Gil-Perotin S, Jurado-Rodriguez A, Candiota AP, DelgadoMederos R, Lope-Piedrafita S, Garcia-Verdugo JM, Arus C, Marti-Fabregas J. In vivo and ex vivo magnetic resonance spectroscopy of the infarct and the subventricular zone in experimental stroke. Journal of cerebral blood flow and metabolism : official journal of the International Society of Cerebral Blood Flow and Metabolism 2015;35(5):828-834. 
39. Scabini M, Stellari F, Cappella P, Rizzitano S, Texido G, Pesenti E. In vivo imaging of early stage apoptosis by measuring real-time caspase-3/7 activation. Apoptosis : an international journal on programmed cell death 2011;16(2):198-207.

40. Kyrylkova K, Kyryachenko S, Leid M, Kioussi C. Detection of apoptosis by TUNEL assay. Methods in molecular biology 2012;887:41-47.

41. Gorin F, Harley W, Schnier J, Lyeth B, Jue T. Perinecrotic glioma proliferation and metabolic profile within an intracerebral tumor xenograft. Acta Neuropathol 2004;107(3):235-244.

42. Noch E, Khalili K. Molecular mechanisms of necrosis in glioblastoma: the role of glutamate excitotoxicity. Cancer Biol Ther 2009;8(19):1791-1797.

43. Raza SM, Lang FF, Aggarwal BB, Fuller GN, Wildrick DM, Sawaya R. Necrosis and glioblastoma: a friend or a foe? A review and a hypothesis. Neurosurgery 2002;51(1):2-12; discussion 12-13.

44. Rong Y, Durden DL, Van Meir EG, Brat DJ. 'Pseudopalisading' necrosis in glioblastoma: a familiar morphologic feature that links vascular pathology, hypoxia, and angiogenesis.

Journal of neuropathology and experimental neurology 2006;65(6):529-539.

45. Hakumaki JM, Grohn OH, Tyynela K, Valonen P, Yla-Herttuala S, Kauppinen RA. Early gene therapy-induced apoptotic response in BT4C gliomas by magnetic resonance relaxation contrast T1 in the rotating frame. Cancer gene therapy 2002;9(4):338-345.

46. Jakubowicz-Gil J, Langner E, Badziul D, Wertel I, Rzeski W. Apoptosis induction in human glioblastoma multiforme T98G cells upon temozolomide and quercetin treatment. Tumour biology : the journal of the International Society for Oncodevelopmental Biology and Medicine 2013;34(4):2367-2378.

47. Tamura K, Wakimoto H, Agarwal AS, Rabkin SD, Bhere D, Martuza RL, Kuroda T, Kasmieh R, Shah K. Multimechanistic tumor targeted oncolytic virus overcomes resistance in brain tumors. Molecular therapy : the journal of the American Society of Gene Therapy 2013;21(1):68-77.

48. Hangel G, Strasser B, Povazan M, Gruber S, Chmelik M, Gajdosik M, Trattnig S, Bogner W. Lipid suppression via double inversion recovery with symmetric frequency sweep for robust 2D-GRAPPA-accelerated MRSI of the brain at $7 \mathrm{~T}$. NMR Biomed 2015;28(11):1413-1425.

49. Zhu H, Ouwerkerk R, Barker PB. Dual-band water and lipid suppression for MR spectroscopic imaging at 3 Tesla. Magn Reson Med 2010;63(6):1486-1492.

50. Ciezka M, Delgado-Goñi T, Ortega-Martorell S, Olier I, Julià-Sape M, Candiota AP, Lisboa PJG, Arús C. Nosological imaging of glioblastoma response to therapy. June 2014; Alcalá de Henares, Spain.

51. Ortega-Martorell S, Olier I, Delgado-Goñi T, Ciezka M, Julià-Sapé M, Lisboa PJG, Arús C. Semi-supervised source extraction methodology for the nosological imaging of glioblastoma response to therapy. IEEE Symposium Series on Computational Intelligence (SSCI). Orlando, FL, USADecember 2014. 
TABLES

TABLE 1

\begin{tabular}{ccccc}
\hline \multicolumn{2}{c}{ TRAINING SET } & \multicolumn{3}{c}{ TEST SET } \\
\hline Control & Treated & Control & \multicolumn{2}{c}{ Treated } \\
\hline C255-day 14 & C415-day 22 & C32-day 16 & C414-day 24 & C526-day 18 \\
C288-day 18 & C418-day 22 & C69-day 15 & C419-day 24 & C574-day 26 \\
C351-day 13 & C437-day 23 & C71-day 16 & C450-day 17 & C572-day 18 \\
C520-day 18 & C525-day 22 & C179-day 17 & C489-day 17 & C776-day 34 \\
C529-day 18 & C527-day 22 & C233-day 17 & C499-day 15 & C795-day 18 \\
C583-day 18 & C575-day 26 & C234-day 17 & C502-day15 & C797-day 22 \\
& C584-day 26 & C278-day 19 & C503-day 15 & C808-day 33 \\
& C586-day 22 & & C521-day 18 & C819-day 45 \\
& & & & C821-day 34
\end{tabular}

TABLE 1: Summary of the 38 animals harboring a GL261 GB included into this work and the day post-inoculation when they were last studied (prior to sacrifice), divided in a training and a test set. The Cxxx notation corresponds to the internal research group unique mouse identifier code. All the animals included were between 18-21 weeks of age and weighted between 20-23 g at the start of the treatment and tumor volumes were not significantly different from $16.9 \pm 6.2 \mathrm{~mm} 3$ (15) .

\section{FIGURES}

FIGURE 1: The profile of the three sources extracted from the cases in the training set is shown in the top row, represented as metabolite relative intensities after UL2 normalization (y-axis) in a frequency range (ppm, $\mathrm{x}$-axis). $\mathrm{R}$ represents the profile of tumor responding to treatment, $\mathrm{T}$ the profile of control/unresponsive tumors and $\mathrm{N}$ is the profile related to normal brain parenchyma. A) The main differences between the responsive and unresponsive patterns and their tentative 
assignments according to literature (see results and discussion sections) are highlighted: higher lactate $(4.1 \mathrm{ppm})$ intensity, combined with lower saturated fatty acids mobile lipids, ML (1.3 ppm), higher total ML (0.9 ppm) and ML polyunsaturated fatty acids (PUFA) (2.8 ppm) resonances and a lower myo-inositol/glycine signal $(3.55 \mathrm{ppm})$ are characteristic from cases responding to TMZ. B) The main differences between normal brain parenchyma and standard GL261 tumors are indicated: higher choline/creatine (3.21/3.03 ppm) ratio, higher mobile lipids (0.9 and $1.3 \mathrm{ppm})$ and lactate (1.3 ppm and $4.1 \mathrm{ppm})$ intensities and lower NAA (2.02 ppm) in the case of the tumoral tissue.

FIGURE 2: A) Three representative cases from the training set (Table 1) are represented, one control (C583) and two treated with 3 TMZ cycles (C418 and C586). From left to right: spectral vectors (labels) selected for training (red represents the tumor label, treated or control, and blue the normal tissue label); nosologic maps obtained for each source with the corresponding color scale on the right that indicates high (red) or low (blue) values in the mixing matrix respectively for each voxel. Next on the right, the resulting nosologic map after combining the three sources, where blue represents normal tissue, green represents responding tissue and red control/unresponsive tumor. All the maps are superimposed over the corresponding $T_{2 w} M R$ image. On the right, representative histopathological samples for each case $\left(0.1 \mathrm{~mm}^{2}\right)$, immunostained with Ki67 and caspase 3 (brownish spots), are displayed (see Table S2 for further details). B) Box-plots representing the reference ranges for proliferation, apoptosis and mitotic indexes calculated for control and treated tumors from (15). Cases from the training set are represented with red (control) and green (treated) stars, with code numbers of cases outside the box-plot reference range incorporated. Note: ${ }^{\circ}$ and $*$ symbols in the boxplot diagrams indicate outliers and extreme outliers, respectively. 
FIGURE 3: Cases included in the training set, control (left) and treated (right), and not represented in Figure 2. From left to right for each case: spectral vectors (labels) selected for training the semi-supervised methodology (red represents the tumor label (treated or control) and blue the normal tissue label); nosologic maps obtained for each source with the corresponding color scale (see Figure 2 for color code); next, the resulting nosologic map after combining the three sources (see Figure 2 for color code). All the maps are superimposed over the corresponding $\mathrm{T}_{2 \mathrm{w}}$ MR image.

FIGURE 4: All the cases included in the test set, control (left) and treated with more than one TMZ cycle (right). Pictures display and color codes as in Figure 3.

FIGURE 5: $T_{2 w}$ reference images and their corresponding nosologic maps superimposed, acquired at selected time-points during and after the three cycles of treatment, in cases C776, C795, C797, C808, C819 and C821 from the test set. For each map, blue represents normal tissue, green represents responding tumor, red control/unresponsive tumor and black undetermined tissue. Under each case there is a color-bar showing the response stage determined by the RECIST criteria along the therapy protocol (red means progressive disease; yellow, stable disease and green partial response).

FIGURE 6: A) Contingency tables correlating the response detected by the semi-supervised methodology and the proliferation and apoptotic indexes in all the cases with apparently homogeneous nosologic maps. B) Proliferation and apoptotic indexes comparison between cases classified as responders or non-responders using the sources analysis. Asterisk (*) labels significant differences. 
A
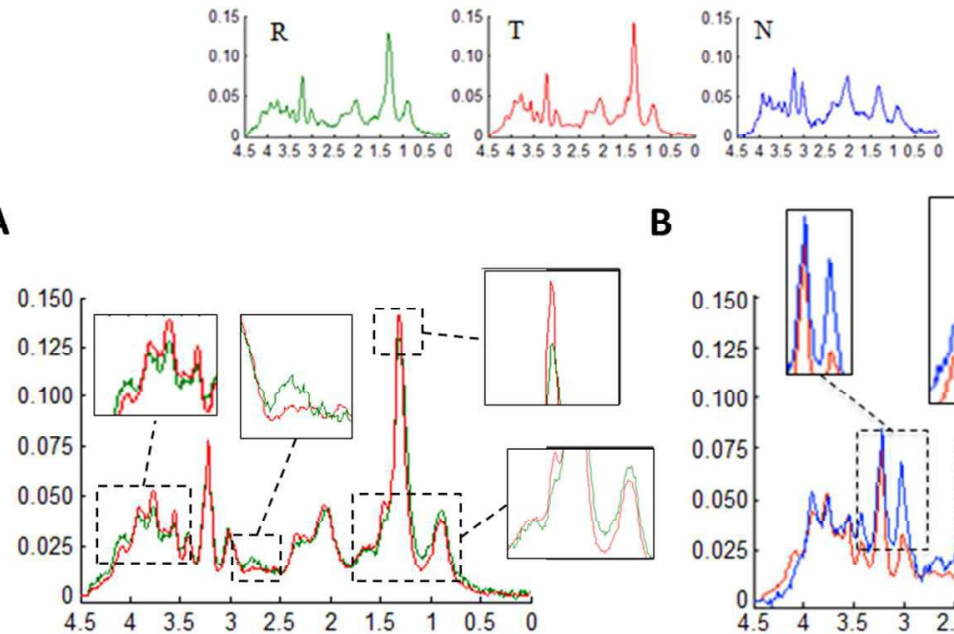

B

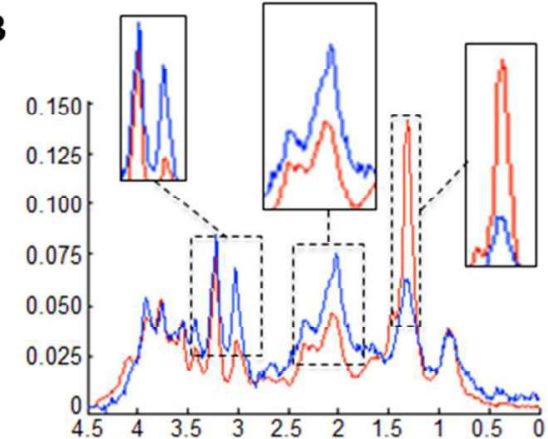

FIGURE 1: The profile of the three sources extracted from the cases in the training set is shown in the top row, represented as metabolite relative intensities after UL2 normalization ( $y$-axis) in a frequency range ( $\mathrm{ppm}, \mathrm{x}$-axis). $\mathrm{R}$ represents the profile of tumor responding to treatment, $\mathrm{T}$ the profile of control/unresponsive tumors and $\mathrm{N}$ is the profile related to normal brain parenchyma. $A$ ) The main differences between the responsive and unresponsive patterns and their tentative assignments according to literature (see results and discussion sections) are highlighted: higher lactate $(4.1 \mathrm{ppm})$ intensity, combined with lower saturated fatty acids mobile lipids, ML $(1.3 \mathrm{ppm})$, higher total $\mathrm{ML}(0.9 \mathrm{ppm})$ and $\mathrm{ML}$

polyunsaturated fatty acids (PUFA) (2.8 ppm) resonances and a lower myo-inositol/glycine signal (3.55ppm) are characteristic from cases responding to TMZ. B) The main differences between normal brain parenchyma and standard GL261 tumors are indicated: higher choline/creatine (3.21/3.03 ppm) ratio, higher mobile lipids $(0.9$ and $1.3 \mathrm{ppm})$ and lactate $(1.3 \mathrm{ppm}$ and $4.1 \mathrm{ppm})$ intensities and lower NAA $(2.02 \mathrm{ppm})$ in the case of the tumoral tissue. 
A

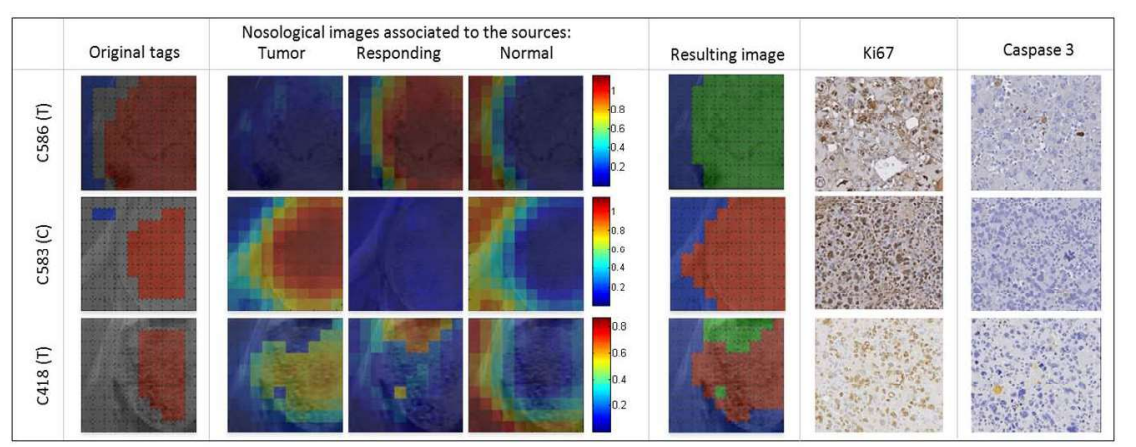

B
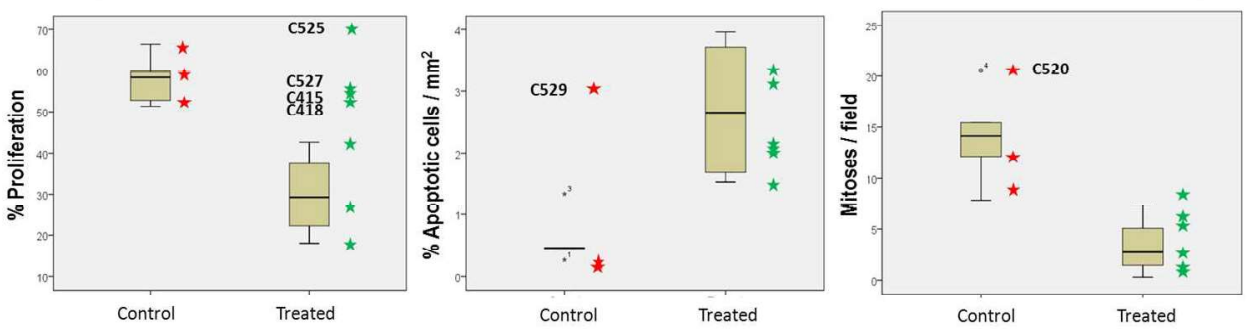

FIGURE 2: A) Three representative cases from the training set (Table 1) are represented, one control (C583) and two treated with 3 TMZ cycles (C418 and C586). From left to right: spectral vectors (labels) selected for training (red represents the tumor label, treated or control, and blue the normal tissue label); nosologic maps obtained for each source with the corresponding color scale on the right that indicates high (red) or low (blue) values in the mixing matrix respectively for each voxel. Next on the right, the resulting nosologic map after combining the three sources, where blue represents normal tissue, green represents responding tissue and red control/unresponsive tumor. All the maps are superimposed over the corresponding T2w MR image. On the right, representative histopathological samples for each case $(0.1 \mathrm{~mm} 2)$, immunostained with

Ki67 and caspase 3 (brownish spots), are displayed (see Table S2 for further details). B) Box-plots representing the reference ranges for proliferation, apoptosis and mitotic indexes calculated for control and treated tumors from (15). Cases from the training set are represented with red (control) and green (treated) stars, with code numbers of cases outside the box-plot reference range incorporated. Note: 0 and * symbols in the boxplot diagrams indicate outliers and extreme outliers, respectively. 

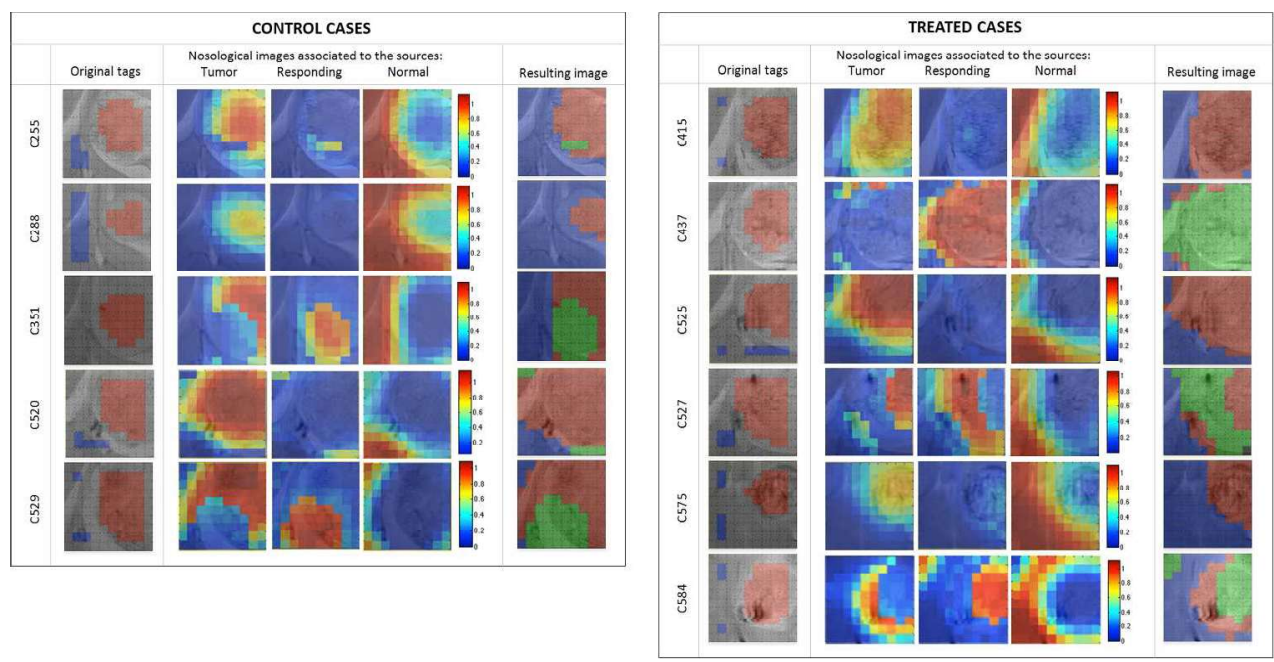

FIGURE 3: Cases included in the training set, control (left) and treated (right), and not represented in Figure 2. From left to right for each case: spectral vectors (labels) selected for training the semi-supervised methodology (red represents the tumor label (treated or control) and blue the normal tissue label); nosologic maps obtained for each source with the corresponding color scale (see Figure 2 for color code); next, the resulting nosologic map after combining the three sources (see Figure 2 for color code). All the maps are superimposed over the corresponding T2w MR image. 

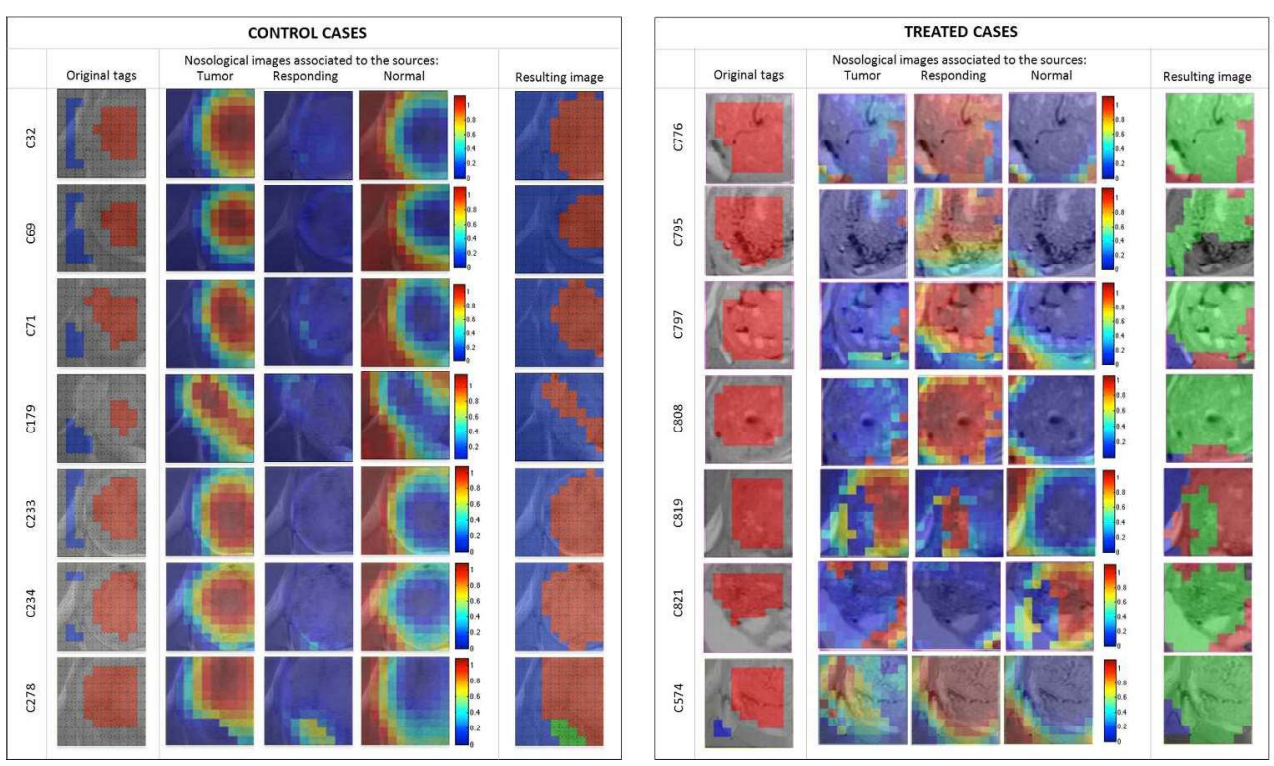

FIGURE 4: All the cases included in the test set, control (left) and treated with more than one TMZ cycle (right). Pictures display and color codes as in Figure 3. 
FIGURE 5: T2W reference images and their corresponding nosologic maps superimposed, acquired at selected time-points during and after the three cycles of treatment, in cases C776, C795, C797, C808, C819 and C821 from the test set. For each map, blue represents normal tissue, green represents responding tumor, red control/unresponsive tumor and black undetermined tissue. Under each case there is a color-bar showing the response stage determined by the RECIST criteria along the therapy protocol (red means progressive disease; yellow, stable disease and green partial response). 
A

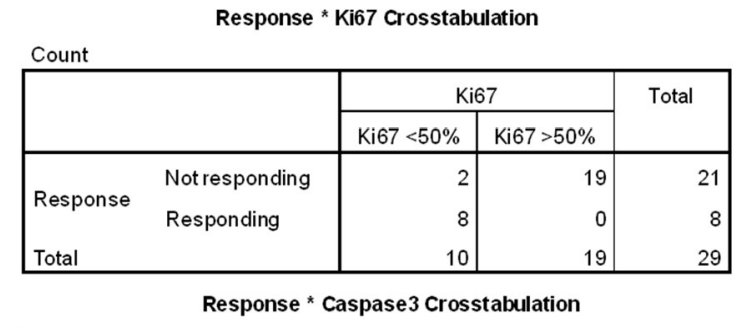

\begin{tabular}{|ll|r|r|r|}
\hline \multirow{2}{*}{ Count } & \multicolumn{2}{|c|}{ Caspase3 } & \multirow{2}{*}{ Total } \\
\cline { 3 - 4 } & & Caspase $<1 \%$ & Caspase $>1 \%$ & \\
\hline \multirow{2}{*}{ Response } & Notresponding & 2 & 12 & 14 \\
& Responding & 4 & 4 & 8 \\
Total & & 6 & 16 & 22 \\
\hline
\end{tabular}

B
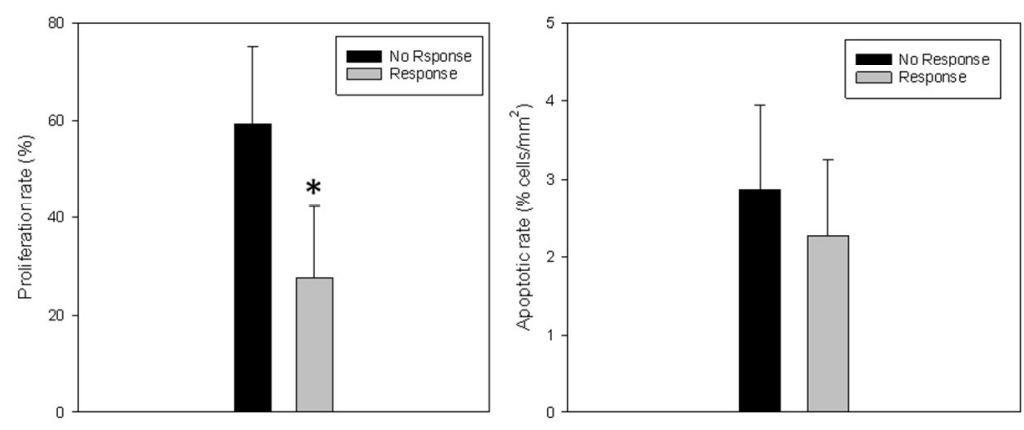

FIGURE 6: A) Contingency tables correlating the response detected by the semi-supervised methodology and the proliferation and apoptotic indexes in all the cases with apparently homogeneous nosologic maps. B) Proliferation and apoptotic indexes comparison between cases classified as responders or non-responders using the sources analysis. Asterisk (*) labels significant differences. 
MRSI-BASED MOLECULAR IMAGING OF THERAPY RESPONSE TO

TEMOZOLOMIDE IN PRECLINICAL GLIOBLASTOMA USING SOURCE

ANALYSIS

Delgado-Goñi T, Ortega-Martorell S, Ciezka M, Olier I, Candiota AP, Julià-Sapé M, Fernández F, Pumarola M, Lisboa PJ, Arús C.

\section{SUPPLEMENTARY METHODS}

\section{In vivo MR studies}

MRI studies

Horizontal, high-resolution $\mathrm{T}_{2 \mathrm{w}}$ images $(\mathrm{TR} / \mathrm{TEeff}=4200 / 36 \mathrm{~ms})$ were acquired using a RARE (Rapid Acquisition with Relaxation Enhancement) sequence (turbo factor, 8; field of view (FOV), $19.2 \times 19.2 \mathrm{~mm}$; matrix, $256 \times 256$ (75 x $75 \mu \mathrm{m} /$ pixel); number of slices, 10; slice thickness (ST), $0.5 \mathrm{~mm}$; inter-slice thickness, $0.6 \mathrm{~mm}$; number of averages (NA), 4; total acquisition time (TAT), 6 min and $43 \mathrm{~s}$, to detect brain tumor presence and its evolution stage.

MRSI studies

A reference $T_{2 \mathrm{~W}}$ high resolution image and a $14 \mathrm{~ms}$ TE MRSI were initially acquired. DMSO was then injected i.p. as in (1) (PE-MRSI), followed by three repeated $14 \mathrm{~ms}$ TE MRSI acquisitions, acquired using a 2D CSI (Chemical Shift Imaging) sequence with PRESS localization, where: FOV, 17.6 x 17.6 mm; Volume of Interest (VOI), (5.5 x 5.5 x $1.0 \mathrm{~mm}$ ), positioned so that most of the tumor and part of the nearby normal/peritumoral brain parenchyma were included; and with ST, 1mm; TR, 2500ms; SW, 4006.41Hz; NA, 512; TAT, $21 \mathrm{~m} 30$ s. Water suppression was performed with 
VAPOR, using a $300 \mathrm{~Hz}$ bandwidth. Linear and second order shims were automatically adjusted with FASTMAP in a $5.8 \times 5.8 \times 5.8 \mathrm{~mm}$ volume which contained the VOI region. Six saturation slices $(\mathrm{ST}, 10 \mathrm{~mm}$; sech-shaped pulses: $1.0 \mathrm{~ms} / 20250 \mathrm{~Hz})$ were positioned around the VOI to minimize outer volume contamination in the signals obtained. Spatial resolution was defined by a $8 \times 8$ voxel matrix over the FOV $(4.84 \mu \mathrm{l}$ nominal resolution) reconstructed after Fourier interpolation to a $32 \times 32$ matrix, as described previously (2).

\section{MR processing}

For most cases, tumor volume was calculated in high resolution horizontal images as follows:

$T V(m m)^{3}=\left[\left(A S_{1}+S T\right)+\left[\left(A S_{2}+(\ldots)+A S_{10}\right) \times(S T+I T)\right]\right] \times 0.075^{2} \quad$ Eq. $[\mathrm{S} 1]$ where TV is the tumor volume, AS is the number of pixels contained in the region of interest delimited by the tumor boundaries in each slice of the MRI sequence, ST is the slice thickness $(0.5 \mathrm{~mm})$, IT the inter-slice thickness $(0.1 \mathrm{~mm})$ and $0.0056 \mathrm{~mm}^{2}$ the individual pixel surface area.

The tumor area was calculated in pixels for each slice, using an automated system for generating regions of interest (ROIs) available in the Paravision 5.0 software (Bruker BioSpin, Ettlingen, Germany). The inter-slice volume was not registered and it was estimated adding the inter-slice thickness to the corresponding slice thickness in Equation S1.

For tumor C584, only a set of low-resolution T2 weighted MRI was available, so the Equation S1 was adjusted accordingly (resolution of $150 \times 150 \mu \mathrm{m} /$ pixel, slice thickness $1 \mathrm{~mm}$, inter-slice thickness $0.1 \mathrm{~mm})$. 
For retrospective control cases (C32, C69, C71, C179, C233, C234 and C278), the volume calculation was performed following a different strategy due to the restricted set of T2 weighted MRI available. Namely, horizontal T2 weighted images were used for an estimation of tumor diameter, both in left-right and feet-head direction. A mean tumor diameter was calculated and used to obtain the radius. Finally, assuming a spherical shape for the tumor, the volume was calculated using the generic sphere volume formula (Equation S2).

$V=\frac{4}{3} \times \pi \times r^{3} \quad$ Eq. [S2]

Where " $r$ " is the calculated average radius of the tumor.

\section{Voxel labeling and Signal source extraction}

The semi-supervised methodology described in (3) was applied for the extraction of meaningful source signals in the tumors from the training set (Figure 1). It proposes to take benefit from the use of prior knowledge derived from class membership of the spectra to guide the source extraction. It involves three main stages (figure S1):

I. The definition of a Fisher Information (FI) metric (4) to model pairwise similarities and dissimilarities between data points, using a MultiLayer Perceptron (MLP) classifier to estimate the conditional probabilities of class membership.

II. The approximation of the empirical data distribution in an Euclidean projective space in which Non-negative Matrix Factorization (NMF)-based techniques can be applied $(5,6)$. They consist on a group of multivariate data analysis techniques aimed to estimate meaningful latent components, also known as sources, from non-negative data. Standard NMF 
methods decompose the data matrix $\mathrm{X}$ into two non-negative matrices $\mathrm{F}$ (the sources) and $H$ (the mixing matrix). The differences between them are given by the different cost functions used for measuring the divergence between $\mathrm{X}$ and $\mathrm{F}^{*} \mathrm{H}$. This is done in the current study with Multidimensional Scaling methods, specifically with the iterative majorization algorithm $(7,8)$.

III. The application of Convex-NMF for the source decomposition of the data. In (9) the authors present a variant of NMF, namely Convex-NMF, in which the basis vectors of $\mathrm{F}$ are constrained to be convex combinations of the data points. Convex-NMF relaxes the NMF constraints to allow negative values both in the data matrix and the sources.

\section{Histopathology}

Representative regions of $0.1 \mathrm{~mm}^{2}$ were selected from the periphery and the core of the tumoral masses, at 20x magnification, using NDPview 1.2.53 software (Hamamatsu Photonics France SARL, Massy, France). The number of selected regions depended on tumor size (between 4 and 17 in the periphery and between 1 and 14 in the core of the tumors). Additional regions were evaluated in selected tumors (e.g. C795). Immunopositive cells for Caspase 3, and positive and negative cells for Ki67 were counted in each field using Image J $1.47 \mathrm{~d}$ software (NIH, USA) and the "Cell Counter" plugin.

The evaluation of necrosis was performed on the HE stained slides. Different features were considered: isolated necrotic cells, moderate amounts of eosynophilic debris and large empty spaces. For each tumor the percentage of the tissue section affected was semi-quantified. When a tumor showed less than $20 \%$ of the mentioned necrotic 
features, a low grade of necrosis was assigned, while high grade of necrosis was assigned to those having more than $20 \%$ of the features.

On the other hand, the number of mitoses in each tumor was counted and evaluated by two neuropathologists in a blind study, in seven selected fields at 40x using an Axioskop 40 microscope (Carl Zeiss, Goettingen, Germany) on the original HE stained slides. See SM below for some specific examples.

\section{SUPPLEMENTARY RESULTS}

\section{Growth curves for GL261 tumors in the training and in the test set}

The volume of 11 cases in the training group (Figure S2A) and 17 cases in the validation group (Figure S2B) was calculated the day when MRSI sequences were acquired, in order to compare the response to therapy described by the sources with a standard response indicator acting as intermediate endpoint biomarker (tumor volume at a certain time point, assessed by MRI), as described in (10), and shown in Figure S2 as average curves. Control/untreated tumors and those treated only with one TMZ cycle show a similar growth curve, significantly different from that of tumors treated with 3 TMZ cycles (10), which shows a characteristic volume growth arrest (stable disease according to RECIST criteria (11)) between days 20 and 28 post-inoculation. The volume for 11 cases in the training set and 17 in the test set was not significantly different from the reference curves described in (10) (see Table S2 and S3 and Figure S2). 
Sensitivity and specificity analysis of tumor/non tumor recognition by source analysis in the training set.

Table S1 compiles the classification accuracies, sensitivity and specificity of the obtained source-based label maps with respect to the labels provided by the experts and used for training (the original labels). For the accuracies, $T$ represents the voxels labeled as tumor, with or without response, and $\mathrm{N}$ represents the non-tumor voxels. For the sensitivity, the true positive (TP) cases are the tumor voxels correctly labeled as tumors (also with or without response), and the false negative (FN) cases are tumor voxels labeled as non-tumor (with or without response). Similarly, for the specificity, the true negative $(\mathrm{TN})$ cases are non-tumor voxels correctly identified as non-tumor, and the false positive (FP) cases are non-tumor voxels (with or without response) labeled as tumor.

Additional histopathology analysis of animals in the training group of the source extraction process.

Analysis of cases displaying heterogeneous nosological image pattern was performed to check for possible local correlation with histopathology. Cases C418 and C527 showed some regions of response according to the nosological imaging, that in a detailed histopathological analysis did not show significant differences with nosologic nonresponding zones ((C418 responding area: $55.4 \pm 3.2 \%$ proliferation, $1.9 \pm 0.06 \%$ apoptotic cells $/ \mathrm{mm}^{2}, \mathrm{C} 418$ non-responding area: $49.3 \pm 5.4 \%$ proliferation , $2.1 \pm 0.1 \%$ apoptotic cells $/ \mathrm{mm}^{2}$ and $2.4 \pm 0.5$ mitoses/field; C527 responding area: $55.5 \pm 14.6 \%$ proliferation, $2.3 \pm 1.7 \%$ apoptotic cells $/ \mathrm{mm}^{2}$, C527 non-responding area: $61.5 \pm 2.6 \%$ proliferation , $1.2 \pm 0.4 \%$ apoptotic cells $/ \mathrm{mm}^{2}$ and $3 \pm 1.4 \mathrm{mitoses} /$ field), despite there 
were more caspase-positive cells in this "responding" region in case C527 and more necrotic tissue in case C418. Case C584, showed a central region of response in the nosological image which would agree with the average values of proliferation, mitotic index and percentage of apoptosis seen in Table S2. The external rim of the tumor (50100 micrometers in the periphery) was sampled and counted for proliferation, apoptosis and mitotic rate $(51.9 \pm 13.2 \% ; 3.2 \%$ and $0.7 \pm 0.1$ respectively), indicating that the external part of the tumor was proliferating (values included in the range for control tumors) and possibly explaining the non-responding voxels detected in the periphery of the nosologic map of this case (Figure 3). Finally, tumor C575, was classified as T by the nosological image in Figure 3, while histopathology of the sampled region (Table S2) would suggest it should be responding. The existence of several masses revealed by the histopathological examination (Figure S5), would suggest that indeed, tumor C575 also contains actively proliferating regions that may have been somewhat captured by the nosological image seen in Figure 3.

\section{Animals in the prospective group of the test set treated with one TMZ cycle}

Ten GL261 cases were treated only with one TMZ cycle. Out of those, all spectral vectors recognized as tumor were also recognized as proliferating tumor, except for 2 out of 27 spectral vectors in C414 and most spectral vectors in C419 (Figure S3), which were found to be responding to treatment. Regarding histopathology, the proliferation indexes for the 6 cases fully recognized as actively proliferating were in the range 52.4 to $79.4 \%$, all equal or significantly higher $(\mathrm{p}<0.05)$ than the average of control untreated tumors $(57.8 \pm 5.4 \%)$ from (10). These high proliferation rates detected by histopathology correlated well with the non-responding pattern detected. On the other hand, in $\mathrm{C} 419$ and $\mathrm{C} 414$ their proliferation rates were significantly lower than the 
average control tumor values (ca. $39 \%$ vs. $57.8 \%$ ) and in one of them (C414), the percentage of apoptotic cells was significantly above the average $(p<0.01)$ of control GL261 tumors (13.3 vs. $0.6 \%$ ). This would be compatible with those two tumors displaying partial response to therapy, being correctly sampled by the nosological image in $\mathrm{C} 419$, while for $\mathrm{C} 414$ there would be a discrepancy between therapy response status proposed by the nosological image and the histopathology data. A further analysis of case $\mathrm{C} 419$ showed that, although the region in the VOI was responding, outside in the periphery (rim of the tumor, see case C795 and figure S8 for an example of rim sampling details) it was actively growing (proliferation $62.5+/-9.4 \%$, apoptotic rate: $0.39 \%$ ), as expected for a tumor treated with only $1 \mathrm{TMZ}$ cycle.

Comparison of proliferation/response/normal parenchyma prediction using the supervised Linear Discriminant Analysis-based classifier described in (10) and the semisupervised source-based classifier from the present work.

The comparison was carried out only in the independent test set cases, unused for classifier development both in (10) and in the present work: the seven untreated control GL261 tumors from (12) and the six mice from the longitudinal part of this work. For the longitudinal cases, only those days when there was an agreement between nosological image and tumor volume after the end of therapy (fulfilling the RECIST criteria for stable disease or response) were included. Figure S6 shows both nosologic maps obtained for the selected cases, either by the LDA-based classifier or the source extraction methodology. A more accurate delimitation of the tumoral mass, according to the abnormal area detected by the $\mathrm{T}_{2 \mathrm{w}}$ images, was obtained using the sources method (as seen for example in case C819 between days 26 to 34 p.i. and control cases C32, 
C69, C81, C179). Only tumor C776 at day 21 was better delimited by the LDA-based nosologic maps regarding the MRI information. Also the voxel classification was more homogeneous in the control retrospective group using the sources methodology, with only one case (C278) showing a responding area inside the tumoral mass, in comparison to the 6 cases with heterogeneous profiles obtained with the LDA-based classifier.

Case C797 at day 18 and case C821 at day 26 were classified as stable disease according to the RECIST criteria and their nosologic maps calculated from the sources showed a $46.2 \%$ and $34.6 \%$ of response and a $53.8 \%$ and $65.4 \%$ of progression respectively, whereas the maps calculated with the LDA method show a $20.5 \%$ and 87.6 $\%$ of response and a $79.5 \%$ and $12.4 \%$ of progression, more related to progressive and responding disease than to the actual status of the tumors, according to RECIST. In conclusion, the sources-based classifier seems more accurate for delimiting tumor boundaries as well as for predicting subsequent tumor response or progression.

\section{Case by case analysis of selected tumors.}

\section{Case C529}

Average proliferation and mitotic indexes for this case were within reference values in (10) for control and treated cases respectively (Figure 2B), but the calculated SD showed heterogeneity between the sampled regions (Table S2). Tumor volume was significantly larger than the reference curve at day 18 . This heterogeneity was reflected in the nosologic map calculated with the sources method (Figure S7B), showing regions of response (green) and progression (red). A more accurate analysis of the two different tumor regions, according to the nosological map, revealed proliferation values of $72.2 \pm 4.2 \%$ and $62.9 \pm 7.2 \%$ in the green and red zones respectively. Also an additional counting performed in the external rim of the tumor $(50-100 \mu \mathrm{m})$ indicated a proliferation rate of $73.9 \pm 7.6 \%$ for this area, an apoptotic rate of $0.74 \%$ and a mitotic rate of $14 \pm 4$ mitoses/field (including both red and green areas 
of the nosologic map). This indicates that the tumor was mostly proliferating in the periphery whereas the central core showed an increased apoptosis (see Figure S7C). The cause for the "response" green zone at the lower left corner of the Figure S7B image remains uncertain.

\section{Case C795}

This mouse died at day 18, before starting the second therapy cycle. The nosological image at day 18 (Figure 4) shows an almost fully responding tumor, in the MRSI sampled ROI, and the average proliferation sample after histopathology would agree with this: $12.6 \%$ (Table S3). Still, the volume of the tumor $\left(152.3 \mathrm{~mm}^{3}\right.$ ) was significantly higher than the average for controls $\left(102 \pm 1.7 \mathrm{~mm}^{3}\right)$, but it remained stable with respect to the previous measurement regarding the RECIST criteria. The explanation to this tumor active growth being missed at day 18 p.i possibly resides in the restricted ROI being sampled by the MRSI, because the high proliferation region of this particular tumor was mostly a thin layer in its periphery (see Figure S8), which was mostly beyond the area evaluated by the usable nosological image.

To analyze this possibility, the $50-100 \mu \mathrm{m}$ rim in the periphery of the tumor was sampled and counted for Ki67, mitosis and apoptosis, in order to compare them with the average tumor mass parameters already counted (Table S3). The proliferation in the outer rim was significantly higher than the value reported in Table S3 $(20.3 \pm 5.8 \%$ in the $50-100 \mu \mathrm{m}$ outer rim versus $12.6 \pm 4.7 \%$ in the average tumor mass). The apoptotic rate in the rim was $0.28 \%$ versus $1 \pm 0.9 \%$ in the average mass. This could partially explain why the animal died so early despite the tumor being recognized as a responder in most of the mass sampled by the nosological map. 


\section{SUPPLEMENTARY FIGURES}

\section{FIGURE S1}

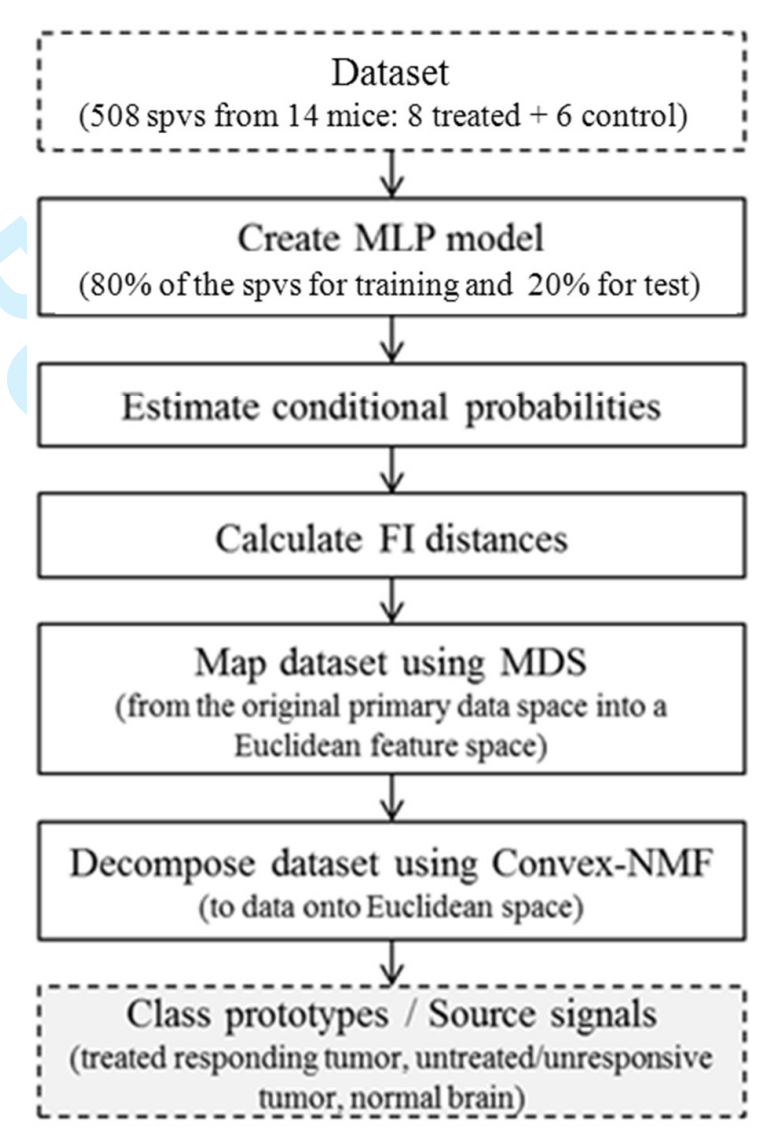

FIGURE S1: Diagram showing the main steps followed in the semi-supervised source extraction methodology, from the collection of MRSI data grids to the generation of the three main sources describing the metabolic profile of the tumors analyzed; spvs, spectral vector elements. 


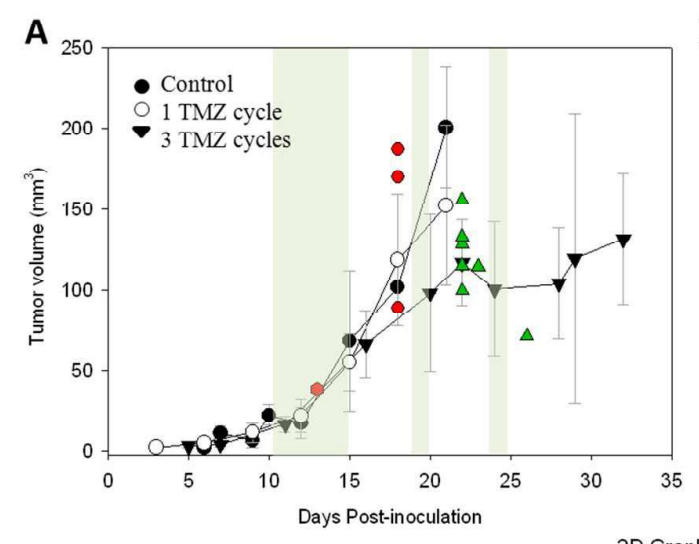

B

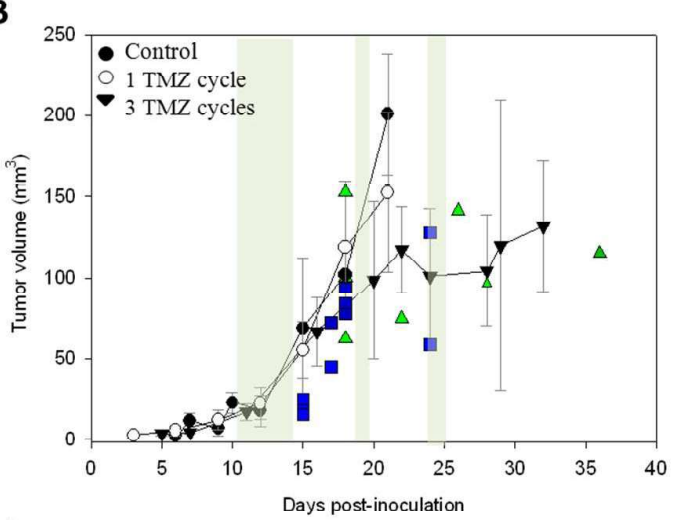

2D Graph 1

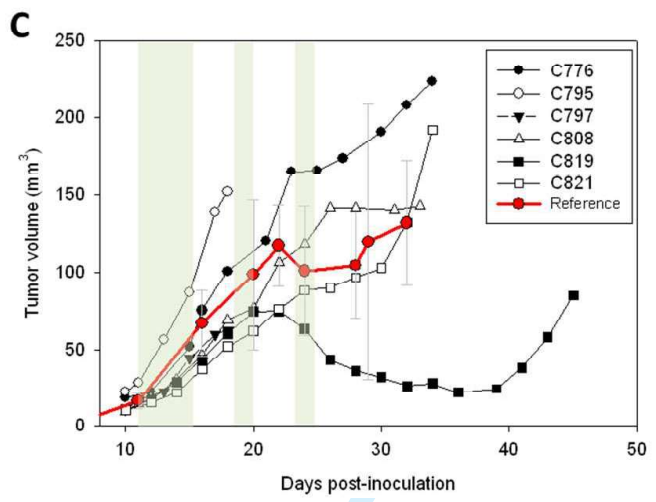

FIGURE S2: In the three sections of this Figure, the average volume ( \pm SD) growth curves of control $(n=6)$ and treated GL261 tumors during the one $(n=8)$ and three $(n=12)$ TMZ cycles protocol (from (10)) are represented for basal reference (note: these are not the same cases quoted in Tables S2 and S3 to provide average proliferation, apoptotic and mitotic indexes). Tumors treated with only one TMZ cycle have a similar growth pattern than control tumors, whereas those treated with three TMZ cycles show a significant volume growth arrest between days 20 and 28 (10). Tumor volumes from control and treated cases in the training and test set from this work were not significantly different to the reference growth curves previously described in (10). A) Tumor volumes available the day of the MRSI scanning for the animals in the training set (see Figure 1 and Table S2) are superimposed to the average curves with red diamonds (control cases) or green "apex up" triangles (treated cases). B) Tumor volume 
representation for 11 treated animals in the test set, treated with one (blue squares) or three TMZ cycles (green "apex up" triangles) for which a single MRSI exploration was available, superimposed in the average curves as in A. C) Tumor growth curves for the six treated animals in the test set that were studied by MRSI at several different timepoints of the therapy protocol. Therapy cycles are highlighted with green shade bars in the three sections of the Figure, while the average reference curve for $3 \mathrm{TMZ}$ cycles treated GL261 bearing-mice from (10) is shown in red in C.

\section{FIGURE S3}

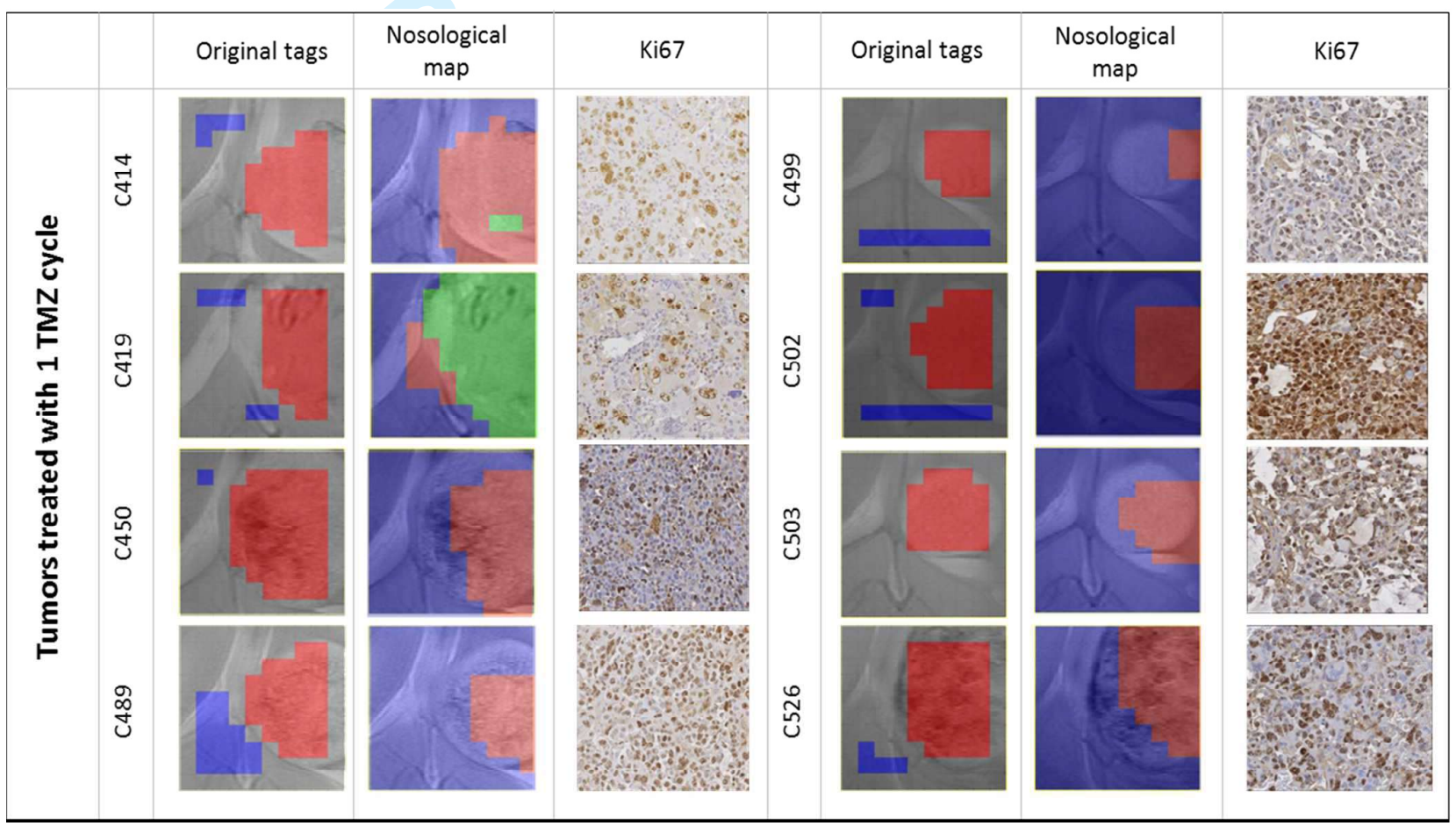

FIGURE S3: Comparison between the combined nosologic maps obtained for the cases included in the test set applying the three sources, (blue for $\mathrm{N}$, green for $\mathrm{R}$ and red for $\mathrm{T}$ ), and a representative field of $0.1 \mathrm{~mm}^{2}$ stained with Ki67. Most of the animals treated with one cycle showed a non-responding profile using the sources methodology, which correlated well with a high proliferation rate detected by Ki67 (Table S2). Case C419 
turned out to be mostly responding to treatment after one cycle, and accordingly, its proliferation index was significantly lower than the average for unresponsive tumors.

\section{FIGURE S4}

A

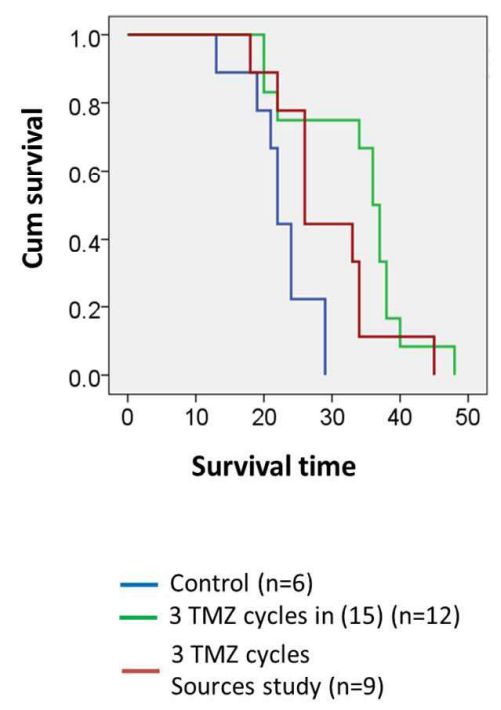

B

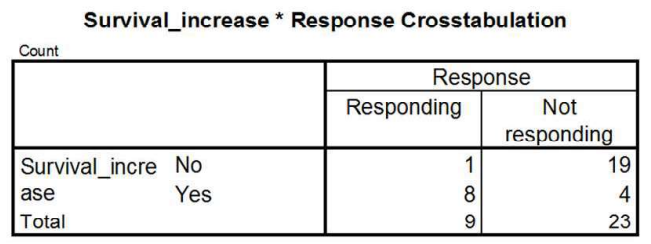

C

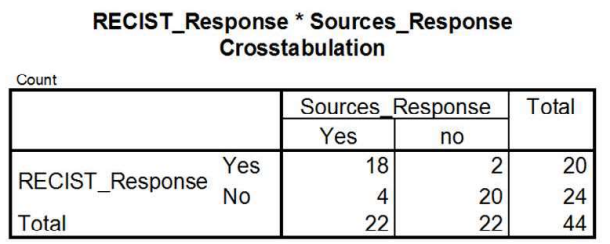

D

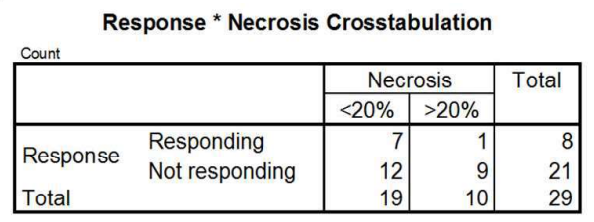

Figure S4: A) Kaplan-Meier survival curves of control GL261 ( $\mathrm{n}=6$, blue) and treated animals with 3 TMZ cycles $(n=12$, green) in (10) and animals treated with 3 TMZ cycles in this work $(n=9$, red). The survival rate observed in this work was not significantly different to the one previously reported for TMZ treated mice. Contingency tables correlating the response detected by the semi-supervised methodology and: B) survival, C) the RECIST response and D) the microscopic necrosis content, considering all the cases with apparently homogeneous nosologic maps. 


\section{FIGURE S5}

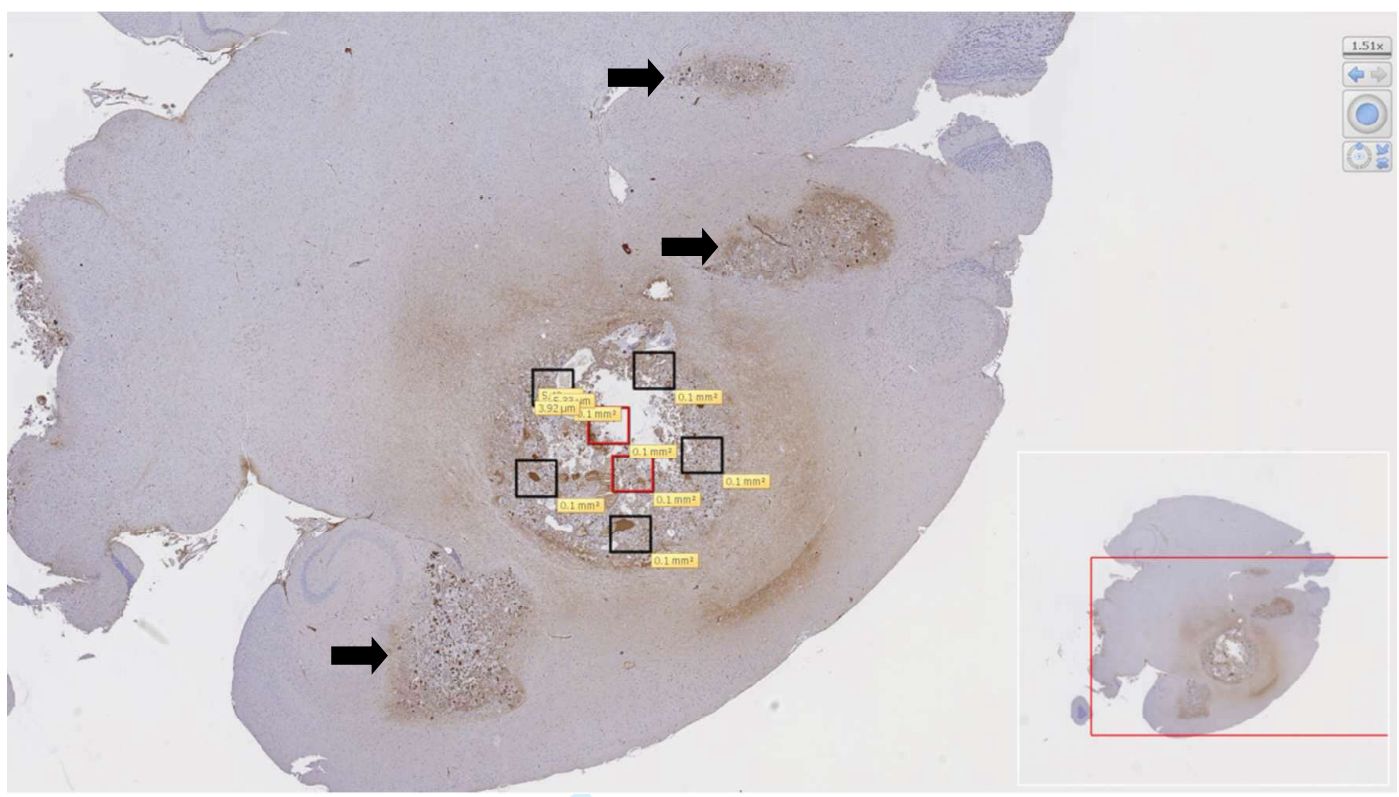

FIGURE S5: Digitized histopathological image of case C575, immunostained for Ki67.

An overview of the whole brain shows that, apart from the main tumoral mass analyzed by MRSI and used for mitotic, apoptotic and proliferation rates calculation, there are three additional tumor foci (marked with black arrows). These masses indicate that despite treatment, the tumor continued to grow in regionally distant areas, with aggressive proliferation, which may have contributed to the spectroscopic pattern detected by the sources methodology. It may well be that the nosological image is somewhat contributed by the proliferation of one of the peripheral masses, while part of the central "responding" mass is seen more as "normal" brain parenchyma. The average proliferation and apoptotic rates in the peripheral tumor masses were: $39.9 \%+/-4.6 \%$ and $1.6 \%$, respectively. 


\section{FIGURE S6}



Figure S6: Treated prospective cases (top) and retrospective control group (bottom) from the test set. For each group, the top row shows the $T_{2 \mathrm{w}}$ MRI VOIs where the MRSI sequences were acquired (time points indicated in the figure), the second row displays the nosologic maps (corresponding to each VOI) calculated using the classification system developed in (10) and the third row shows the nosologic maps calculated with the sources methodology (see Figure S3 for the color legend). 


\section{FIGURE S7}

A

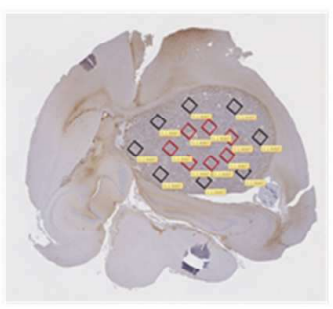

C520

C

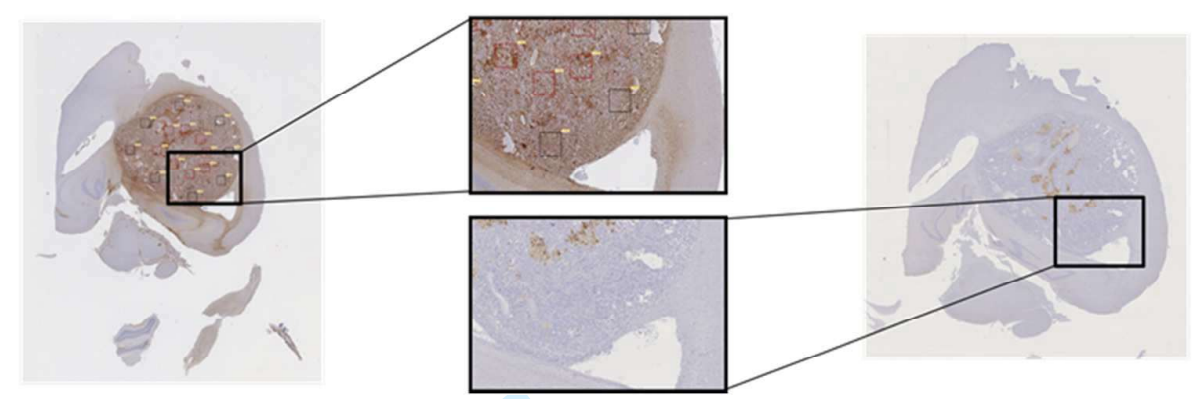

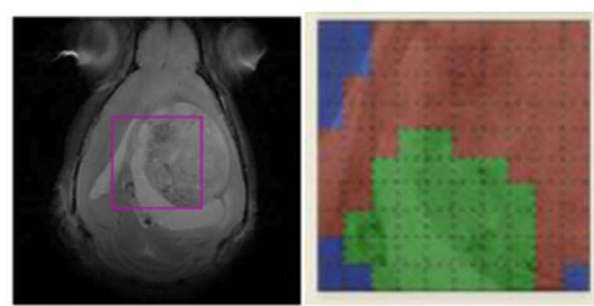

C586

Figure S7: Examples of typical histopathology sampling for control (C520) and responding cases (C586) are shown in A. Case C529 heterogeneous nosologic map is displayed in B, and representative regions where a more accurate sampling for Ki67 (top, central column) and caspase 3 (bottom, central column) was performed (external rim) are shown in $\mathrm{C}$.

\section{FIGURE S8}



\section{TABLE S1}

\begin{tabular}{|l|l|l|l|}
\hline Mouse & Accuracies & Sensitivity & Specificity \\
\hline C415 & T: $100 \%(31 / 31), \mathrm{N}: 100 \%(2 / 2)$ & 1 & 1 \\
\hline C418 & T: $93.1 \%(27 / 29), \mathrm{N}:-$ & 0.93 & - \\
\hline C437 & T: $100 \%(29 / 29), \mathrm{N}:-$ & 1 & - \\
\hline C525 & T: $100 \%(26 / 26), \mathrm{N}: 67 \%(4 / 6)$ & 1 & 1 \\
\hline C527 & T: $100 \%(40 / 40), \mathrm{N}: 100 \%(4 / 4)$ & 1 & 1 \\
\hline C575 & T: $94.7 \%(18 / 19), \mathrm{N}: 100 \%(5 / 5)$ & 0.95 & 1 \\
\hline C584 & T: $100 \%(31 / 31), \mathrm{N}: 66.7 \%(2 / 3)$ & 1 & 0.67 \\
\hline C586 & T: $98.6 \%(71 / 72), \mathrm{N}: 100 \%(13 / 13)$ & 0.99 & 1 \\
\hline C255 & T: $100 \%(26 / 26), \mathrm{N}: 100 \%(6 / 6)$ & 1 & 1 \\
\hline C288 & T: $76.9 \%(10 / 13), \mathrm{N}: 100 \%(16 / 16)$ & 0.77 & 1 \\
\hline C351 & T: $100 \%(25 / 25), \mathrm{N}:-$ & 1 & - \\
\hline C520 & T: $100 \%(31 / 31), \mathrm{N}: 100 \%(5 / 5)$ & 1 & 1 \\
\hline C529 & T: $100 \%(37 / 37), \mathrm{N}: 0 \%(0 / 3)$ & 1 & 0 \\
\hline C583 & T: $100 \%(34 / 34), \mathrm{N}: 100 \%(2 / 2)$ & 1 & 1 \\
\hline Mean & T: $98.4 \%(436 / 443), \mathrm{N}: 90.8 \%(59 / 65)$ & 0.97 & 0.88 \\
\hline
\end{tabular}

Table S1.

T represents tumor, with or without response. $\mathrm{N}$ represents non-tumor.

The last row contains the mean values of each column.

Note: In the cases of C418, C437, and C351 the specificity cannot be calculated because there were no non-tumor labels fulfilling predefined inclusion criteria for those mice. 


\section{TABLE S2}

\begin{tabular}{|c|c|c|c|c|c|c|c|}
\hline & & & $\begin{array}{c}\text { MRSI } \\
\text { acquisition } \\
\text { (day post- } \\
\text { inoculation) }\end{array}$ & $\begin{array}{c}\text { Tumor volume } \\
\qquad\left(\mathrm{mm}^{3}\right)^{*}\end{array}$ & $\begin{array}{c}\text { Proliferation rate } \\
(\%)\end{array}$ & $\begin{array}{l}\text { Apoptotic rate } \\
\left(\% \text { cells } / \mathbf{m m}^{2}\right)\end{array}$ & $\begin{array}{l}\text { Mitotic index } \\
\text { (mitoses/field) }\end{array}$ \\
\hline \multirow{3}{*}{\multicolumn{2}{|c|}{ Control }} & $\mathrm{C520}$ & 18 & 187.5 & $51.4 \pm 12.2$ & $0.46 \pm 0.4$ & $20.6 \pm 7.9$ \\
\hline & & C529 & 18 & 170 & $66.5 \pm 15.5$ & $2.8 \pm 2.8$ & $7.9 \pm 7.2$ \\
\hline & & C583 & 18 & 89.4 & $57.9 \pm 13.1$ & $0.5 \pm 0.3$ & $12.1 \pm 3.6$ \\
\hline \multicolumn{3}{|c|}{$\begin{array}{l}\text { Average in }(10) \\
\qquad N=6\end{array}$} & 18 & $102 \pm 1.7$ & $\mathbf{5 7 . 8} \pm \mathbf{5 . 4}$ & $0.6 \pm 0.4$ & $14.1 \pm 4.2$ \\
\hline \multirow{7}{*}{ Treated } & C415 & $2 \mathrm{TMZ}$ cycles & 22 & 115.4 & $53.6 \pm 12.7$ & $1.9 \pm 0.9$ & $4.9 \pm 1.8$ \\
\hline & C418 & $2 \mathrm{TMZ}$ cycles & 22 & 156.2 & $51.7 \pm 12.9$ & $2.1 \pm 1$ & $5.6 \pm 1.9$ \\
\hline & C525 & $2 \mathrm{TMZ}$ cycles & 22 & 100 & $69.9 \pm 6.5$ & $1.8 \pm 0.9$ & $0.8 \pm 0.5$ \\
\hline & C527 & $2 \mathrm{TMZ}$ cycles & 22 & 128.9 & $55.7 \pm 13.7$ & $2.2 \pm 1.4$ & $7.7 \pm 4.3$ \\
\hline & C575 & $3 \mathrm{TMZ}$ cycles & 26 & 71.7 & $18 \pm 5.7$ & $1.5 \pm 0.8$ & $0.3 \pm 2.3$ \\
\hline & C584 & $3 \mathrm{TMZ}$ cycles & 26 & 59.8 & $26.4 \pm 10.2$ & $3.5 \pm 1.4$ & $2.9 \pm 2.3$ \\
\hline & C586 & $2 \mathrm{TMZ}$ cycles & 22 & 133.3 & $43.8 \pm 14.1$ & $3.3 \pm 2.5$ & $1.6 \pm 0.9$ \\
\hline \multicolumn{3}{|c|}{$\begin{array}{c}\text { Average in }(10) \\
N=4\end{array}$} & 24 & $100.9 \pm 41.6$ & $29.8 \pm 10.3$ & $2.7 \pm 1.2$ & $3.3 \pm 2.9$ \\
\hline
\end{tabular}

TABLE S2: Values calculated in some of the training set cases $(n=10)$ for tumor volume (acquired from T2w MRI) and histopathological parameters: proliferation (Ki67+), apoptotic (caspase 3) and mitotic rates. All values are compared with the corresponding averages for GL261 control and treated GB (average values for the whole tumor) reported in (10). Tumor volume from control and treated cases was not significantly different to the reference growth curves described in (10). 
TABLE S3

\begin{tabular}{|c|c|c|c|c|c|c|c|}
\hline & & & $\begin{array}{c}\text { MRSI } \\
\text { acquisition } \\
\text { (day post- } \\
\text { inoculation) }\end{array}$ & $\begin{array}{c}\text { Tumor volume } \\
\qquad\left(\mathrm{mm}^{3}\right)^{*}\end{array}$ & $\begin{array}{c}\text { Proliferation } \\
\text { rate }(\%)\end{array}$ & $\begin{array}{l}\text { Apoptotic rate } \\
\left(\% \text { cells } / \mathbf{m m}^{2}\right)\end{array}$ & $\begin{array}{l}\text { Mitotic index } \\
\text { (mitoses/field) }\end{array}$ \\
\hline \multirow{7}{*}{\multicolumn{2}{|c|}{ Control }} & C32 & 16 & 51.0 & $59.9 \pm 15.8$ & n.a. & $13.8 \pm 3.7$ \\
\hline & & C69 & 15 & 33.5 & $63.5 \pm 12.5$ & n.a. & $21.7 \pm 4.4$ \\
\hline & & C71 & 16 & 35.2 & $63.8 \pm 10.7$ & n.a. & $22.1 \pm 4.6$ \\
\hline & & C179 & 17 & 66.4 & $51.1 \pm 8$ & n.a. & $21.8 \pm 7.2$ \\
\hline & & $\mathrm{C} 233$ & 17 & 64.5 & $63.9 \pm 10.4$ & n.a. & $15.9 \pm 5.4$ \\
\hline & & $\mathrm{C} 234$ & 17 & 71.5 & $59.6 \pm 7$ & n.a. & $16.6 \pm 3.5$ \\
\hline & & $\mathrm{C} 278$ & 19 & 162.8 & $58.1 \pm 10.6$ & n.a. & $6.1 \pm 0.7$ \\
\hline \multicolumn{2}{|c|}{$\begin{array}{c}\begin{array}{c}\text { Average in (10) } \\
\mathrm{N}=6\end{array} \\
\end{array}$} & & 18 & $102 \pm 1.7$ & $\mathbf{5 7 . 8} \pm 5.4$ & $0.6 \pm 0.4$ & $14.1 \pm 4.2$ \\
\hline \multirow{13}{*}{ Treated } & C414 & $1 \mathrm{TMZ}$ cycle & 24 & 62.3 & $39.4 \pm 8.5$ & $13.3 \pm 5.1$ & $2.3 \pm 0.8$ \\
\hline & C419 & $1 \mathrm{TMZ}$ cycle & 24 & 127.7 & $39.1 \pm 8.9$ & $2.3 \pm 0.9$ & $7 \pm 5.8$ \\
\hline & C450 & $1 \mathrm{TMZ}$ cycle & 17 & 65.6 & $52.4 \pm 14$ & $2.8 \pm 3.1$ & $10.3 \pm 5.8$ \\
\hline & C489 & $1 \mathrm{TMZ}$ cycle & 17 & 40.7 & $77.1 \pm 18.4$ & $3.2 \pm 1.4$ & $3 \pm 2.7$ \\
\hline & C499 & $1 \mathrm{TMZ}$ cycle & 15 & 16.8 & $69.2 \pm 17.2$ & $1.7 \pm 1.1$ & $3.3 \pm 2.4$ \\
\hline & $\mathrm{C502}$ & $1 \mathrm{TMZ}$ cycle & 15 & 33.7 & $79.4 \pm 20.6$ & $2.9 \pm 0.9$ & $8.7 \pm 2.1$ \\
\hline & C503 & $1 \mathrm{TMZ}$ cycle & 15 & 23.1 & $59.9 \pm 15.1$ & $1.8 \pm 0.9$ & $3.1 \pm 1.1$ \\
\hline & C521 & $1 \mathrm{TMZ}$ cycle & 18 & 79.2 & $66.6 \pm 15.3$ & $2.1 \pm 1.3$ & $6.9 \pm 2.2$ \\
\hline & $\mathrm{C526}$ & 1 TMZ cycle & 18 & 80.8 & $64.4 \pm 14.1$ & $1.2 \pm 0.6$ & $4.9 \pm 3$ \\
\hline & C572 & $1 \mathrm{TMZ}$ cycle & 18 & 66.1 & $69.8 \pm 18.3$ & $2.4 \pm 1.5$ & $3.1 \pm 1.7$ \\
\hline & C574 & $3 \mathrm{TMZ}$ cycles & 26 & 72.8 & $32.2 \pm 12$ & $3.9 \pm 1.9$ & $2.6 \pm 2.2$ \\
\hline & C776 & $3 \mathrm{TMZ}$ cycles & 34 & 223.5 & $23.7 \pm 5.2$ & $0.5 \pm 0.4$ & $0.9 \pm 0.9$ \\
\hline & C795 & $1 \mathrm{TMZ}$ cycle & 18 & 152.3 & $12.6 \pm 4.7$ & $1 \pm 0.9$ & $1.3 \pm 0.9$ \\
\hline
\end{tabular}




\begin{tabular}{rlccccc}
$\mathbf{C 7 9 7}$ & 2 TMZ cycles & 22 & 96.9 & $41.4 \pm 8.9$ & $0.7 \pm 0.7$ & $2.3 \pm 1.4$ \\
$\mathbf{C 8 0 8}$ & 3 TMZ cycles & 33 & 142.6 & $20.1 \pm 8.6$ & $0.5 \pm 0.5$ & $1.3 \pm 2.1$ \\
C821 & 3 TMZ cycles & 34 & 192.5 & $28.9 \pm 7.5$ & $0.7 \pm 0.6$ & $2.1 \pm 1.1$ \\
\hline $\begin{array}{c}\text { Average in (10) } \\
\mathbf{N = 4}\end{array}$ & & $\mathbf{2 4}$ & $\mathbf{1 0 0 . 9} \pm \mathbf{4 1 . 6}$ & $\mathbf{2 9 . 8} \pm \mathbf{1 0 . 3}$ & $\mathbf{2 . 7 0} \pm \mathbf{1 . 2}$ & $\mathbf{3 . 3} \pm \mathbf{2 . 9}$
\end{tabular}

TABLE S3: Tumor volume (acquired from T2w MRI) and histopathological parameters (proliferation, apoptotic and mitotic rates) calculated for the test set cases. All the values are compared with the corresponding averages for control and treated GB (average values for the whole tumor) reported in (10). ${ }^{*}$ Tumor volume from control and treated cases was not significantly different to the reference growth curves described in (10). 


\section{SUPPLEMENTARY REFERENCES}

1. Delgado-Goni T, Martin-Sitjar J, Simoes RV, Acosta M, Lope-Piedrafita S, Arus C. Dimethyl sulfoxide (DMSO) as a potential contrast agent for brain tumors. NMR in biomedicine 2013;26(2):173-184.

2. Simoes RV, Delgado-Goni T, Lope-Piedrafita S, Arus C. 1H-MRSI pattern perturbation in a mouse glioma: the effects of acute hyperglycemia and moderate hypothermia. NMR in biomedicine 2010;23(1):23-33.

3. Ortega-Martorell S, Ruiz H, Vellido A, Olier I, Romero E, Julia-Sape M, Martin JD, Jarman IH, Arus C, Lisboa PJ. A novel semi-supervised methodology for extracting tumor type-specific MRS sources in human brain data. PLoS One 2013;8(12):e83773.

4. Amari S. Information geometry on hierarchy of probability distributions. IEEE Trans. Inf. Theory. Volume 47; 2001. p 1701-1711.

5. Lee DD, Seung HS. Learning the parts of objects by non-negative matrix factorization. Nature 1999;401(6755):788-791.

6. Paatero P, Tapper U. Positive matrix factorization: A non-negative factor model with optimal utilization of error estimates of data values. Environmetrics 1994;5(2):111-126.

7. Heiser WJ. Convergent computation by iterative majorization: Theory and applications in multidimensional data analysis, in: Recent Advances in descriptive Multivariate Analysis, W. J. Krzanowski. Oxford: Oxford University Press; 1995. p 157-189.

8. Kiers HAL. Setting up alternating least squares and iterative majorization algorithms for solving various matrix optimization problems. Comput Stat Data Anal 2002;41(1):157-170.

9. Ding C, Li T, Jordan MI. Convex and semi-nonnegative matrix factorizations. IEEE Trans Pattern Anal Mach Intell 2010;32(1):45-55.

10. Delgado-Goni T, Julia-Sape M, Candiota AP, Pumarola M, Arus C. Molecular imaging coupled to pattern recognition distinguishes response to temozolomide in preclinical glioblastoma. NMR in biomedicine 2014;27(11):1333-1345.

11. Eisenhauer EA, Therasse P, Bogaerts J, Schwartz LH, Sargent D, Ford R, Dancey J, Arbuck S, Gwyther S, Mooney M, Rubinstein L, Shankar L, Dodd L, Kaplan R, Lacombe D, Verweij J. New response evaluation criteria in solid tumours: revised RECIST guideline (version 1.1). Eur J Cancer 2009;45(2):228247.

12. Ortega-Martorell S, Lisboa PJ, Vellido A, Simoes RV, Pumarola M, Julia-Sape $\mathrm{M}$, Arus C. Convex Non-Negative Matrix Factorization for Brain Tumor Delimitation from MRSI Data. PLoS One 2012;7(10):e47824. 